\title{
Threshold Dynamics for Networks with Arbitrary Surface Tensions
}

\author{
SELIM ESEDOḠLU \\ University of Michigan \\ FELIX OTTO \\ Max Planck Institute for Mathematics in the Sciences
}

\begin{abstract}
We present and study a new algorithm for simulating the $N$-phase mean curvature motion for an arbitrary set of (isotropic) $\frac{N(N-1)}{2}$ surface tensions. The departure point is the threshold dynamics algorithm of Merriman, Bence, and Osher for the two-phase case.

A new energetic interpretation of this algorithm allows us to extend it in a natural way to the case of $N$ phases, for arbitrary surface tensions and arbitrary (isotropic) mobilities. For a large class of surface tensions, the algorithm is shown to be consistent in the sense that at every time step, it decreases an energy functional that is an approximation (in the sense of Gamma convergence) of the interfacial energy. A broad range of numerical tests shows good convergence properties.

An important application is the motion of grain boundaries in polycrystalline materials: It is also established that the above-mentioned large class of surface tensions contains the Read-Shockley surface tensions, both in the twodimensional and three-dimensional settings. (C) 2015 Wiley Periodicals, Inc.
\end{abstract}

\section{Introduction}

We present and study new algorithms for simulating the mean curvature motion of networks of interfaces under arbitrary surface tensions. This motion arises as the $L^{2}$ gradient descent for an energy in which the area of each surface in the network is weighted by a possibly different constant. It appears prominently in several fields including materials science, where it describes the motion of grain boundaries in polycrystalline materials [30], and in computer vision, where it is used for segmenting images [31].

The mathematical setting of the problem is as follows: Let $D$ be a domain in $\mathbb{R}^{d}$, typically $d=3$. For convenience, we will work mostly on a cube with periodic boundary conditions, so that $D$ will in fact be a torus. Consider a partition of $D$ into closed sets $\Sigma_{1}, \ldots, \Sigma_{N}$ called phases that may intersect only through their 


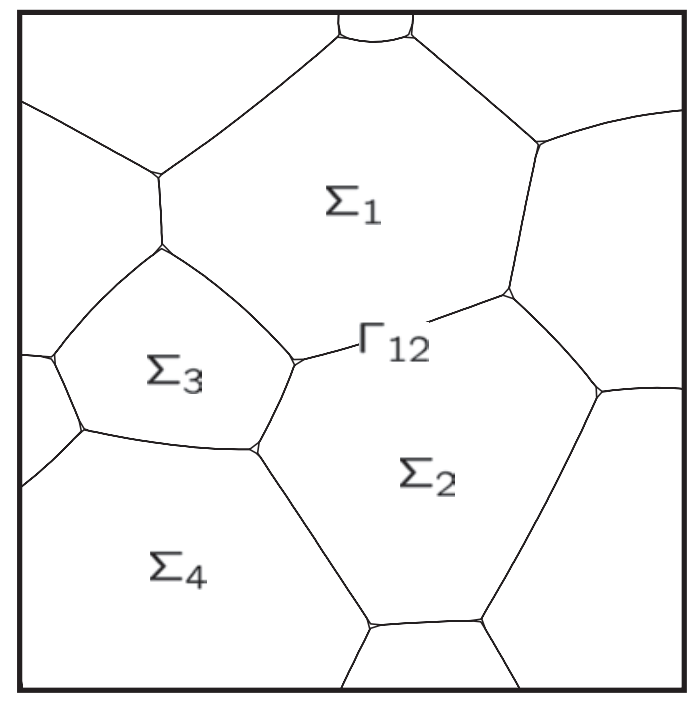

FIGURE 1.1. A partitioning of a domain into sets $\Sigma_{j}$ that intersect only at their boundaries. Interface $\Gamma_{i, j}$ separates $\Sigma_{i}$ from $\Sigma_{j}$. This set-up appears e.g. in materials science, where the sets $\Sigma_{j}$ represent grains (individual single-crystal pieces) making up a polycrystalline material.

boundaries:

$$
D=\bigcup_{j=1}^{N} \Sigma_{j} \quad \text { and } \quad \Sigma_{i} \cap \Sigma_{j}=\left(\partial \Sigma_{i}\right) \cap\left(\partial \Sigma_{j}\right) \quad \text { for } i \neq j .
$$

Denote the interface separating $\Sigma_{i}$ and $\Sigma_{j}$ by $\Gamma_{i, j}$ :

$$
\Gamma_{i, j}=\left(\partial \Sigma_{i}\right) \cap\left(\partial \Sigma_{j}\right) .
$$

See Figure 1.1 for an illustration.

The energy we study, defined on partitions of $D$, is

$$
E\left(\Sigma_{1}, \ldots, \Sigma_{N}\right)=\sum_{i, j=1}^{N} \sigma_{i, j} \operatorname{Area}\left(\Gamma_{i, j}\right)
$$

where $\sigma_{i, i}=0$ and $\sigma_{i, j}=\sigma_{j, i}$ are strictly positive for $i \neq j$. The constant $\sigma_{i, j}$ is called the surface tension associated with interface $\Gamma_{i, j}$. Denote the set of surface tension matrices as

$$
\mathcal{S}_{N}=\left\{\sigma \in \mathbb{R}^{N \times N}: \sigma_{i, i}=0 \text { and } \sigma_{i, j}=\sigma_{j, i}>0 \text { for all distinct } i, j\right\} .
$$

The following triangle inequality is necessary and sufficient for model (1.2) to be well-posed (lower semicontinuous) [29]:

$$
\sigma_{i, j}+\sigma_{j, k} \geq \sigma_{i, k} \quad \text { for any } i, j, \text { and } k .
$$


We will therefore work mostly with the triangle-inequality-satisfying class of surface tensions:

$$
\mathcal{T}_{N}=\left\{\sigma \in \mathcal{S}_{N}: \sigma_{i, j}+\sigma_{j, k} \geq \sigma_{i, k} \text { for any } i, j, k\right\} .
$$

Junctions are locations in the domain $D$ of the partition where more than two grains meet. For a given partition, it is convenient to (informally) define the following sets of points in discussing junctions:

$$
\mathcal{J}_{k}=\left\{x \in D: \exists \varepsilon>0 \text { s.t. } B_{r}(x) \text { intersects } k \text { phases } \forall r<\varepsilon\right\},
$$

so that

$$
\mathcal{J}_{1}=\bigcup_{i=1}^{N} \stackrel{\circ}{\Sigma}_{i}, \quad \mathcal{J}_{\geq 3}=\{\text { Junctions }\}, \quad \text { and } \quad \mathcal{J}_{2}=\left(\bigcup_{i \neq j} \Gamma_{i, j}\right) \backslash \mathcal{J}_{\geq 3} .
$$

Our goal is to develop efficient, robust algorithms for simulating the $L^{2}$ gradient flow of energy (1.2). Two important rules defining the resulting dynamics are:

(1) At any point $p \in \Gamma_{i, j} \backslash \mathcal{J}_{\geq 3}$ at which $\Gamma_{i, j}$ is smooth, the normal speed is given by

$$
v_{\perp}(p)=\mu_{i, j} \sigma_{i, j} \kappa_{i, j}(p)
$$

where $\kappa_{i, j}$ denotes the mean curvature of $\Gamma_{i, j}$. The constants $\mu_{i, j}$ are the mobilities associated with the interfaces $\Gamma_{i, j}$; they are positive, but otherwise can be chosen arbitrarily.

(2) A condition known as the Herring angle condition [18] holds at triple junctions $p \in \mathcal{J}_{3}$ : At a junction formed by the meeting of the three phases $\Sigma_{1}$, $\Sigma_{2}$, and $\Sigma_{3}$, one has

$$
\sigma_{1,2} n_{1,2}(p)+\sigma_{2,3} n_{2,3}(p)+\sigma_{3,1} n_{3,1}(p)=0
$$

where $n_{i, j}$ denotes the unit normal to $\Gamma_{i, j}$, pointing from $\Sigma_{i}$ into $\Sigma_{j}$. Relation (1.6) determines the opening angles $\theta_{1}, \theta_{2}$, and $\theta_{3}$ (see Figure 1.2) of the three phases $\Sigma_{1}, \Sigma_{2}$, and $\Sigma_{3}$, respectively, in terms of the surface tensions:

$$
\frac{\sin \theta_{1}}{\sigma_{2,3}}=\frac{\sin \theta_{2}}{\sigma_{1,3}}=\frac{\sin \theta_{3}}{\sigma_{1,2}} .
$$

These two rules do not completely specify the dynamics, because topological changes in the network of surfaces inevitably take place, and multiple junctions (where four or more phases meet) routinely form even if absent in the initial condition. There is, in fact, no complete theory of solutions for this system covering all possible types of junctions and surviving past topological changes. Rather, these two are necessary conditions to be met by any reasonable algorithm. Additional necessary conditions can be derived, e.g., at stable multiple (>3) junctions [7].

We conclude this introduction with an outline of the paper:

- Section 2 describes the motivation from materials science for studying the dynamics considered in this paper. 


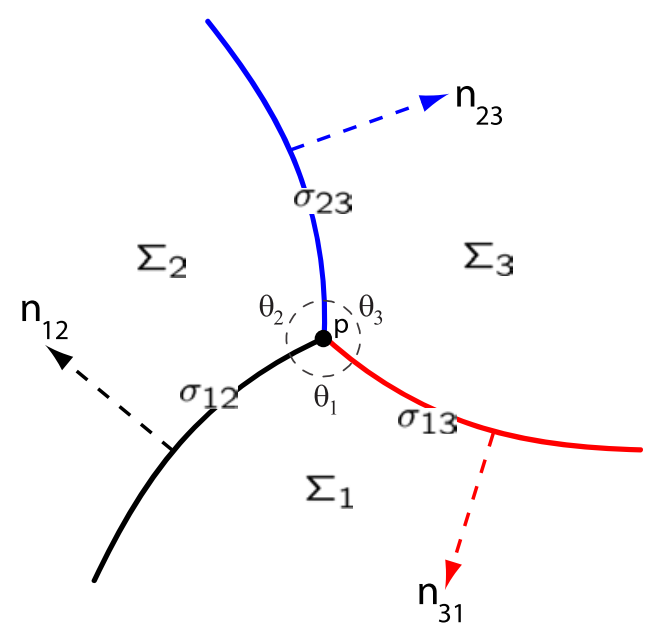

FIGURE 1.2. The angles $\theta_{j}$ formed at a triple junction $p$ are determined according to formula (1.7) by the surface tensions $\sigma_{i, j}$ of the interfaces meeting there. The tangent to the triple curve points into the page in this picture.

- Section 3 recalls important previous work, in particular the original threshold dynamics algorithm of Merriman, Bence, and Osher [26,27] that constitutes the departure point of the algorithms presented here. It also describes difficulties in extending this algorithm to the general setting of arbitrary surface tensions that is the focus of the present work.

- Section 4 presents an energetic formulation of threshold dynamics. In particular, it identifies a class of approximations to surface energy that constitute Lyapunov functionals for threshold-dynamics-type algorithms. This is new even in the case of the most basic, two-phase setting. More importantly, it reveals a principled way of extending threshold dynamics to the setting of the general interfacial energy (1.2).

- Section 5.1 contains the derivation of the main result of the paper: Our threshold dynamics algorithm for general surface tensions $\sigma_{i, j}$ and arbitrary mobilities $\mu_{i, j}$, fully described in 5.26-5.29. Section 5.2 studies stability properties of the proposed algorithm and establishes its unconditional gradient stability in a wide class of cases. This matter turns out to be related to certain well-known questions of embeddability of finite metric spaces in euclidean spaces that arises in theoretical computer science and combinatorics. Among the cases covered by our stability result is the physically relevant case of Read-Shockley surface energies, both in the two-dimensional and three-dimensional crystallography settings. Section 5.3 makes connections between our algorithm and the minimizing movements approach, which may prove useful in the future study of convergence 
of the dynamics. Section 5.4 presents a slightly more costly version of our threshold dynamics algorithm, the stability of which applies much more widely to essentially all physically relevant cases.

- Section 6 presents numerical evidence, including both classical convergence studies for smooth flows and experiments with a number of interesting singular phenomena such as topological changes, wetting, and nucleation.

- Finally, the Appendix contains a rigorous proof of the Gamma convergence of our approximate energies to the interfacial energy (1.2). Since the allimportant Herring angle condition (1.7) is an equilibrium (energetic) condition, this result constitutes strong indication for the correct behavior of our algorithm at triple junctions.

\section{Motivation}

Energy (1.2) and its dynamics (1.5) and (1.7) arise in materials science, where they describe the motion of grain boundaries in polycrystals [30] under annealing (heat treatment). A material is called polycrystalline if it is composed of many tiny single crystal pieces, known as grains, stuck together. These types of materials are very common: they include most metals and ceramics. Connected components of the phases $\Sigma_{i}$ in model (1.2) represent individual grains. The surface tension $\sigma_{i, j}$ associated with the interface $\Gamma_{i, j}$ between two neighboring grains $\Sigma_{i}$ and $\Sigma_{j}$ depends on the mismatch between the crystallographic orientations of $\Sigma_{i}$ and $\Sigma_{j}$ [20]. In reality, grain boundary energy also depends on the normal $n_{i, j}$ to the interface $\Gamma_{i, j}$. Here we will ignore this dependence on the normal.

Certain important physical properties of polycrystalline materials, such as their yield strength and conductivity, depend on their grain boundary network. It is therefore of interest to simulate the evolution of grain boundaries under common industrial processes. In certain cases, it is found that the dependence of the energy density in (1.2) on misorientation is fairly constant for large enough misorientations. In such cases, the simplest case of model (1.2) with all equal surface tensions $\sigma_{i, j}=1$ (which leaves no dependence on the specific type of material) provides a reasonable description of some of the grain boundary motion phenomena observed in experiments. However, for certain important grain phenomena, such as the evolution of the grain boundary character distribution [22], the full generality of model (1.2), where each $\sigma_{i, j}$ can be different, is required at the bare minimum.

In [10-12], a version of diffusion-generated motion that is more accurate on uniform grids (relying on signed distance functions as opposed to characteristic functions to represent phases) was used to carry out large-scale simulations of grain growth and recrystallization in three dimensions, but only in the equal surface tension case. In this paper, we develop algorithms so that such large-scale simulations can be carried out for the full generality of model 1.2 so that phenomena such as the grain boundary character distribution can be studied via diffusion generated motion. 


\section{Previous Work}

There is a large body of work on algorithms for simulating the curvature motion (1.5) and (1.7) of interfacial networks; see e.g. [21,23] and references therein for a glimpse of this extensive landscape. The algorithms proposed in the present paper are motivated by the diffusion generated motion scheme of Merriman, Bence, and Osher (MBO) introduced in [26, 27]. The essential idea there is to represent the phases $\Sigma_{i}$ via their characteristic functions $\mathbb{1}_{\Sigma_{i}}$ and generate the desired motion of their boundaries by alternating two simple operations: (1) convolution with a positive, unit mass, radially symmetric kernel $G$ such as the Gaussian

$$
G_{\delta t}(x)=\frac{1}{(4 \pi(\delta t))^{d / 2}} e^{-\frac{|x|^{2}}{4(\delta t)}}
$$

and (2) thresholding. To be precise, the original Merriman-Bence-Osher scheme can be written as follows:

$$
\begin{aligned}
& \text { Algorithm (MBO'92): Given the partition } \Sigma_{1}^{k}, \ldots, \Sigma_{N}^{k} \text { at time } \\
& t=(\delta t) k \text {, to get the partition } \Sigma_{1}^{k+1}, \ldots, \Sigma_{N}^{k+1} \text { at the next time step } \\
& t=(\delta t)(k+1) \text { : } \\
& \quad \text { 1. Convolution step: } \\
& \qquad \phi_{i}^{k}=G_{\delta t} * \mathbb{1}_{\Sigma_{i}^{k}} .
\end{aligned}
$$

2. Thresholding (redistribution) step:

$$
\Sigma_{i}^{k+1}=\left\{x: \phi_{i}^{k}(x)>\phi_{j}^{k}(x) \text { for all } j \neq i\right\} .
$$

The algorithm is appealing because it appears to be unconditionally stable, and each of its steps can be implemented efficiently on a uniform grid: at $M \log (M)$ cost per time step where $M$ is the total number of grid points. Numerical experiments presented in [27] (and subsequently in [34] with an improved implementation on adaptive grids) yield ample empirical evidence for the convergence of this algorithm to the dynamics (1.5) and (1.7) with symmetric $\left(120^{\circ}\right)$ angles at triple junctions. In other words, it appears to generate the gradient flow of energy (1.2) with equal surface tensions:

$$
\sigma_{i, j}=1 \quad \text { for all } i \neq j .
$$

A natural idea for extending the MBO scheme to the general surface tensions case of model (1.2), where each $\sigma_{i, j}$ can be different, is to replace the thresholding (redistribution) step (3.3) of the standard MBO scheme with a weighted version:

$$
\Sigma_{i}^{k+1}=\left\{x: \sum_{\ell} \alpha_{i, \ell} \phi_{\ell}(x)>\sum_{\ell} \alpha_{j, \ell} \phi_{\ell}(x) \text { for all } j \neq i\right\} .
$$

The essential question is then how the constants $\alpha_{i, j}$ should be chosen to induce the desired angles at junctions. 

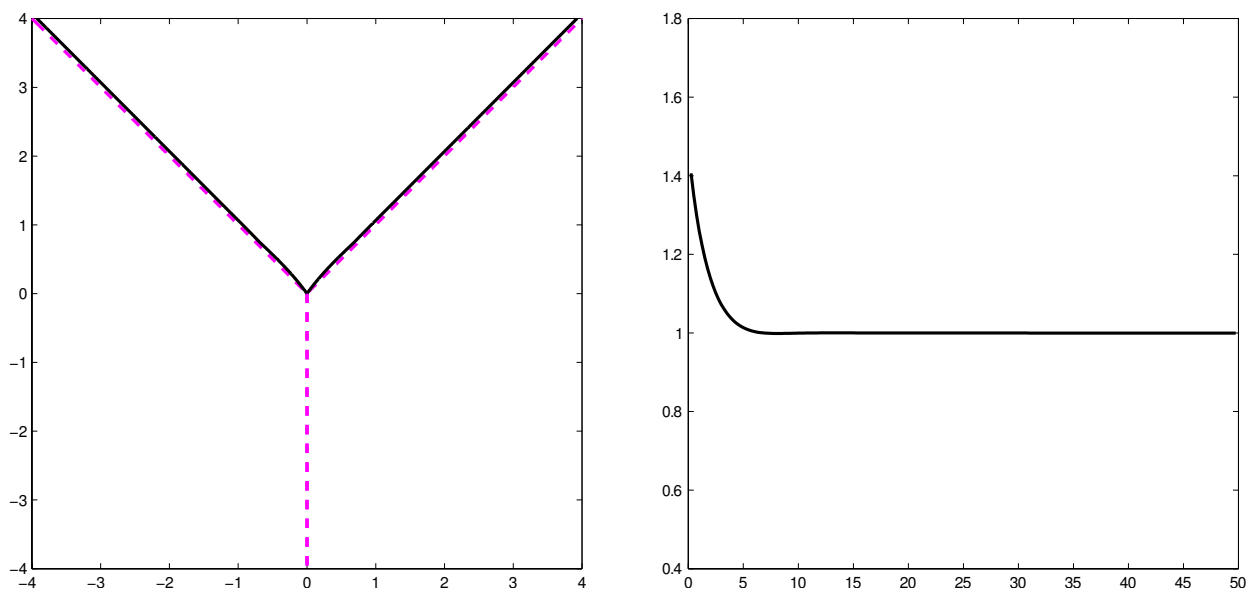

FIGURE 3.1. Left: Solid curves represent a fixed point of our proposed threshold dynamics algorithm, with $\left(90^{\circ}, 90^{\circ}, 120^{\circ}\right)$ angles at the junction. The dotted lines are the exact stationary solution of dynamics (1.5) and (1.7). Right: Zooming in on the junction and plotting the slope of one of the interfaces as a function of distance from the junction makes the presence of a boundary layer (of thickness $\approx \sqrt{\delta t}$ ) evident. These boundary layers were not recognized in several previous attempts at designing threshold dynamics algorithms, and consequently prevented them from achieving the proper angle conditions (1.7). The bottom line: The effective and thus relevant angle is the far-field angle, not the angle at the junction. Section 5 shows how to choose parameters in the proposed algorithm so that the desired effective angle is achieved.

Although several ideas, including a redistribution similar to (3.4), for extending the MBO scheme to the general surface tensions case are proposed in the original paper [27], these do not achieve the correct Herring angle conditions (1.7) at junctions. Related ideas for the same goal appear in the well-known but unpublished notes [25]; these, too, are incorrect.

One difficulty responsible for these failed attempts appears to be the presence of boundary layers in stationary states of MBO-type schemes when unequal surface tensions are attempted using modified redistribution rules such as 3.4. Figure 3.1 shows the boundary layers at the junction for a stationary state of a generalized MBO scheme of the form (3.4). Previous attempts in [25,27] can be understood as trying to impose the Herring angle condition (1.7) right at the junction, oblivious to the presence of boundary layers. The relevant, effective junction angles, however, are not the ones formed right at the junction, but the far-field angles between the interfaces, which are asymptotically straight at an intermediate-length scale. One of the contributions of the present paper is a systematic procedure for determining the effective junction angles induced by any given set of parameters in generalized 
threshold dynamics schemes. Our approach avoids having to understand in detail the structure of the boundary layer.

In [33], a variant of the MBO scheme is proposed that replaces the thresholding step (3.3) by a spatially dependent one. The convolutions formed in step (3.2) are used to estimate the distance of a given point to the nearest triple junction, which is then utilized in assigning the point to one of the phases via a modified thresholding step. Extensive numerical tests in [33] indicate that this modification indeed allows the algorithm to achieve the Herring angle conditions at triple junctions. However:

(1) The resulting algorithm-in particular, its thresholding step-is considerably more complicated than the original MBO scheme, and deviates from its spirit by having to essentially locate triple junctions.

(2) To treat the full generality of model (1.2), a heuristic averaging step is introduced that requires taking a weighted sum over $N$-choose- 3 ways of redistributing points, drastically increasing computational cost.

(3) Since the scheme is designed around triple junctions, there is only some empirical evidence for its behavior when multiple junctions (where four or more phases meet) inevitably form during the evolution.

In contrast, in Section 5 we provide algorithms for the full generality of model (1.2), allowing any triangle-inequality-satisfying choice of surface tensions $\sigma_{i, j}$. These algorithms maintain the simplicity, efficiency, and spirit of the original MBO scheme, and thus appear to be its correct generalization to the unequal surface tension setting. The Appendix and 5.2 present rigorous results strongly indicating that our algorithms automatically impose the appropriate Herring condition according to formula (1.7) at any triple junction. Careful numerical convergence studies in Section 6 provide further evidence.

\section{The Approximate Energies}

This section studies an approximation to the weighted surface area functional (1.2) that turns out to be a Lyapunov functional for the original threshold dynamics scheme and our subsequent extensions of it. This is new even in the case of two-phase threshold dynamics, which had previously been rigorously studied only via comparison principles [5, 13]. The two-phase version of the approximate energy appears in previous literature [1]. Interestingly, these two-phase energies also appear in recent nonlocal models of aggregation and swarming in biological systems [38,39]. Gamma convergence of such nonlocal energies to the perimeter of sets is also established [1]. Extension of these nonlocal approximations of perimeter to the multi-phase energy (1.2), discussed below in this section, is new and allows us to identify in a systematic manner threshold-dynamics-type schemes for (1.2) in Section 5 . 


\subsection{Notation and the New Multiphase Approximation}

In [1, 28], the following approximation for the perimeter of a set $\Sigma \in \mathbb{R}^{N}$ is considered:

$$
P_{\delta t}(\Sigma)=\frac{1}{\sqrt{\delta t}} \int_{\Sigma^{\mathrm{c}}} G_{\delta t} * \mathbb{1}_{\Sigma} d x
$$

In words: An initially uniform temperature distribution in the set $\Sigma$ is allowed to be diffused by the heat equation. Measuring the amount of heat that escapes to the exterior of the set gives, after normalization, an estimate for the size of its boundary; hence the term "heat content." A slightly more general nonlocal approximation to the perimeter of sets is studied in [1]; one of its results is

$$
\Gamma-\lim _{\delta \rightarrow 0^{+}} P_{\delta t}(\Sigma)=\operatorname{Per}(\Sigma) .
$$

For certain choices of the surface tension matrix $\sigma \in \mathcal{T}_{N}$, energy 1.2 can be written as a positive sum of perimeters of sets, and thus the Gamma convergence result of [1] can be directly extended to the multiphase setting for such surface tensions. However, as discussed at length in Section 5.2, energy (1.2) cannot be written as a positive sum of perimeters of sets for all $\sigma \in \mathcal{T}_{N}$. We therefore look for a more general approximation of (1.2) that is in the same spirit as (4.1).

The idea is to approximate the surface area of interface $\Gamma_{i, j}$ appearing in energy (1.2) by the term

$$
\operatorname{Area}\left(\Gamma_{i, j}\right) \approx \frac{1}{\sqrt{\delta t}} \int \mathbb{1}_{\Sigma_{i}} G_{\delta t} * \mathbb{1}_{\Sigma_{j}} d x
$$

which has the intuitive interpretation that the surface area of the interface $\Gamma_{i, j}$ that separates $\Sigma_{i}$ from $\Sigma_{j}$ is related to the amount of heat that escapes from $\Sigma_{j}$ into $\Sigma_{i}$. Thus our approximation to model (1.2) has the form

$$
E_{\delta t}\left(\Sigma_{1}, \ldots, \Sigma_{N}\right)=\frac{1}{\sqrt{\delta t}} \sum_{i, j=1}^{N} \sigma_{i, j} \int \mathbb{1}_{\Sigma_{i}} G_{\delta t} * \mathbb{1}_{\Sigma_{j}} d x
$$

Alternatively, the energy can also be approximated as

$$
\widetilde{E}_{\delta t}\left(\Sigma_{1}, \ldots, \Sigma_{N}\right)=\frac{1}{\sqrt{\alpha_{i, j} \delta t}} \sum_{i, j=1}^{N} \sigma_{i, j} \int \mathbb{1}_{\Sigma_{i}} G_{\alpha_{i, j} \delta t} * \mathbb{1}_{\Sigma_{j}} d x
$$

which involves a convolution with a different kernel for each interface. This is inconvenient from a numerical perspective. Moreover, unlike (4.2), the Gamma convergence of which is studied in the Appendix, convergence for (4.3) is not clear. In this paper we will therefore focus on the approximation 4.2. 
The relaxation of energy (4.2) over functions $u_{j}$ taking their values in the unit interval $[0,1]$ (as opposed to in $\{0,1\}$ ) and adding up to 1, i.e.,

$$
u_{i} \geq 0 \text { and } \sum_{i=1}^{N} u_{i}=1 \quad \text { almost everywhere, }
$$

will also be denoted by $E_{\delta t}$ :

$$
E_{\delta t}\left(u_{1}, \ldots, u_{N}\right)=\frac{1}{\sqrt{\delta t}} \sum_{i, j=1}^{N} \sigma_{i, j} \int u_{i} G_{\delta t} * u_{j} d x .
$$

Also, let the vector $u$ denote $u(x)=\left(u_{1}(x), \ldots, u_{N}(x)\right)$; we will then write $E_{\delta t}(u)$ in place of (4.5). Configurations $u$ respecting (4.4) will be called admissible.

We conclude this section by mentioning that in the context of phase field models, an energy analogous to (4.2) appears in [15,17].

\section{The New Algorithm}

Section 5.1 presents a heuristic derivation of the proposed algorithm for (1.5) and (1.7) for arbitrary mobilities and surface tensions. Section 5.2 provides justification.

\subsection{Derivation of the Algorithm}

The proposed algorithm for (1.5) and (1.7) will be derived as a peculiar optimization procedure for approximate surface energies 4.2) or 4.3). As a special case, this general discussion will also exhibit the original MBO scheme (3.2) and (3.3) as an optimization procedure (for the particular case of equal surface tensions, $\sigma_{i, j}=1$ for all $i \neq j$ ), which is a new characterization.

\section{Relaxed and Linearized Energies}

Denote the set of binary functions $u=\left(u_{1}, \ldots, u_{N}\right)$ on $D$ as

$$
\mathcal{B}=\left\{u \text { : for each } x \text { there is } i \text { s.t. } u_{i}(x)=1 \text { and } u_{j}(x)=0 \text { for all } j \neq i\right\} \text {. }
$$

Binary functions $u$ thus represent characteristic functions of the partitions $\Sigma=$ $\left(\Sigma_{1}, \ldots, \Sigma_{N}\right)$ in (1.1) over which (4.5) agrees with the original approximate energy (4.2). We consider minimizing energy (4.5) over the following convex set $\mathcal{K}$ of functions instead:

$$
\mathcal{K}=\left\{u: u_{j}(x) \in[0,1] \text { for all } x \text { and } j, \text { and } \sum_{j=1}^{N} u_{j}(x)=1 \text { for all } x\right\}
$$

i.e., $\mathcal{K}$ is the set of admissible configurations $(4.4)$. It is a relaxation of the nonconvex constraint set $\mathcal{B}$. The following lemma establishes the equivalence of minimizing (4.5) over the convex constraint set $\mathcal{K}$ and minimizing the original approximate 
energy (4.2). It is stated with the addition of a linear term to 4.5 ) to prevent triviality of the minimizer.

LEMMA 5.1. Let $\sigma \in \mathcal{S}_{N}$ and the convolution kernel $G_{\delta t}$ be the Gaussian kernel (3.1). Let $L$ be any linear functional defined on $\mathcal{K}$. Then

$$
\min _{u \in \mathcal{B}}\left(E_{\delta t}(u)+L(u)\right)=\min _{u \in \mathcal{K}}\left(E_{\delta t}(u)+L(u)\right) .
$$

Proof. Let $v \in \mathcal{K}$ be the minimizer of $E_{\delta t}+L$ on $\mathcal{K}$. Suppose $v \notin \mathcal{B}$. Then there exists a set $A \subset D$ of positive measure, an $\varepsilon>0$, and $k, \ell \in\{1,2, \ldots, N\}$ with $k \neq \ell$ such that

$$
v_{k}(x), v_{\ell}(x) \in(\varepsilon, 1-\varepsilon) \quad \text { for all } x \in A .
$$

Consider the competitor

$$
u_{m}(x, t)=v_{m}(x, t)+t\left(\delta_{m, \ell}-\delta_{m, k}\right) \mathbb{1}_{A}(x)
$$

for $m=1,2, \ldots, N$. Then we have $\sum_{m} u_{m}(x, t)=1$ and $u_{m}(x, t) \geq 0$ for $t \in(-\varepsilon, \varepsilon)$ so that $u(\cdot, t) \in \mathcal{K}$ for $t$ in that range. We have

$$
\frac{d}{d t} u_{m}(x, t)=\left(\delta_{m, \ell}-\delta_{m, k}\right) \mathbb{1}_{A}(x)
$$

and so

$$
\begin{aligned}
\frac{d^{2}}{d t^{2}} E_{\delta t}(u(x, t)) & =2 \sum_{m, n} \sigma_{m, n} \int\left(\frac{d}{d t} u_{m}\right)\left(G_{\delta t} * \frac{d}{d t} u_{n}\right) d x \\
& =2 \sum_{m, n} \sigma_{m, n}\left(\delta_{m, \ell}-\delta_{m, k}\right)\left(\delta_{n, \ell}-\delta_{n, k}\right) \int \mathbb{1}_{A} G_{\delta t} * \mathbb{1}_{A} d x \\
& =2\left(\sigma_{\ell, \ell}-\sigma_{\ell, k}-\sigma_{k, \ell}+\sigma_{k, k}\right) \int \mathbb{1}_{A} G_{\delta t} * \mathbb{1}_{A} d x \\
& =-4 \sigma_{\ell, k} \int \mathbb{1}_{A} G_{\delta t} * \mathbb{1}_{A} d x \\
& <0
\end{aligned}
$$

Thus, $v(x)=u(x, 0)$ cannot be a minimizer.

Let $\mathcal{L}_{E_{\delta t}}\left(u^{k}, \cdot\right)$ denote, up to terms constant in $u$, the linearization of (4.5) at $u^{k}=\left(u_{1}^{k}, \ldots, u_{N}^{k}\right)$ :

$$
\begin{aligned}
\mathcal{L}_{E_{\delta t}}\left(u^{k}, u\right) & =\frac{1}{\sqrt{\delta t}} \sum_{i, j=1}^{N} \sigma_{i, j} \int u_{i} G_{\delta t} * u_{j}^{k}+u_{j} G_{\delta t} * u_{i}^{k} d x \\
& =\frac{2}{\sqrt{\delta t}} \sum_{i=1}^{N} \int u_{i}\left(\sum_{j \neq i} \sigma_{i, j} G_{\delta t} * u_{j}^{k}\right) d x .
\end{aligned}
$$


Denote the coefficient of $u_{i}$ in the integrand of (5.2) as

$$
\phi_{i}^{k}:=\sum_{j=1}^{N} \sigma_{i, j} G_{\delta t} * u_{j}^{k}
$$

so that 5.2 can be written succinctly as

$$
\mathcal{L}_{E_{\delta t}}\left(u^{k}, u\right)=\frac{2}{\sqrt{\delta t}} \sum_{i=1}^{N} \int u_{i} \phi_{i}^{k} d x .
$$

\section{Algorithm for Mobilities $\mu_{i, j}=1 / \sigma_{i, j}$}

It turns out that threshold-dynamics-type schemes for $(1.5)$ and $(1.7)$ can be systematically derived from the approximate energies (4.2) via the following peculiar optimization strategy for the relaxed version (4.5) of (4.2):

$$
u^{k+1}=\underset{\mathrm{u} \in \mathcal{K}}{\arg -\min } \mathcal{L}_{E_{\delta t}}\left(u^{k}, u\right)
$$

In words: at each iteration, the linearization (5.4) of energy (4.5) is minimized over the entire constraint set (5.1). Since (5.5) consists of minimizing a linear functional over the simplex $\mathcal{K}$, the extreme points of which are $\mathcal{B}$, the solution $u^{k+1}$ can always be taken to be binary (i.e., $u^{k+1} \in \mathcal{B}$ ).

Since optimization problem (5.5) involves the minimization of a linear, pointwise functional over a convex constraint set, it is easily solved: The minimization can be carried out at each $x \in D$ independently, upon which the solution is found by comparing the coefficients $\phi_{i}^{k}(x)$ of $u_{i}(x)$ in the integrand of (5.4): At time step $k+1$, the point $x$ belongs to the phase whose coefficient is smallest at $x$ :

$$
u_{i}^{k+1}(x)= \begin{cases}1 & \text { if } \phi_{i}^{k}(x)=\min _{\ell} \phi_{\ell}^{k}(x) \\ 0 & \text { otherwise }\end{cases}
$$

with the proviso that ties of the type $\phi_{i}^{k}(x)=\phi_{j}^{k}(x)=\min _{\ell} \phi_{\ell}^{k}(x)$ for $i \neq j$ can be broken by insisting that $u_{i}^{k+1}(x)=1 \Rightarrow u_{j}^{k+1}(x)=0$ if $j<i$.

Update procedure (5.6) is the analogue of the standard thresholding step of the MBO scheme and extends it to arbitrary surface tensions for an arbitrary number of phases. In fact, (5.6) reduces to the basic thresholding criterion of MBO in the equal surface tension (i.e., $\sigma_{i, j}=1$ for all $i \neq j$ ) case. It is, however, different from all previous attempts at generalizing the MBO thresholding scheme to arbitrary surface tensions. A complete description of the algorithm is as follows: 
Algorithm: Given the initial partition $\Sigma_{1}^{0}, \ldots, \Sigma_{N}^{0}$ with $\Sigma_{i}^{0}=\left\{x: \psi_{i}^{0}(x)>0\right\}$, to obtain the partition $\Sigma_{1}^{k+1}, \ldots, \Sigma_{N}^{k+1}$ at time step $t=(\delta t)(k+1)$ from the partition $\Sigma_{1}^{k}, \ldots, \Sigma_{N}^{k}$ at time $t=(\delta t) k$ :

1. Convolution step: Compute the following convolutions:

$$
\phi_{i}^{k}=G_{\delta t} *\left(\sum_{j=1}^{N} \sigma_{i, j} \mathbb{1}_{\Sigma_{j}^{k}}\right) .
$$

2. Thresholding (redistribution) step:

$$
\Sigma_{i}^{k+1}=\left\{x: \phi_{i}^{k}(x)<\min _{j \neq i} \phi_{j}^{k}(x)\right\} .
$$

From (5.8), a level set function delineating the boundary of grain $i$ at the end of time step $k$ can be formed as follows:

$$
\psi_{i}^{k+1}(x)=\min _{\ell \neq i} \phi_{\ell}^{k}(x)-\phi_{i}^{k}(x)
$$

We then have

$$
\Sigma_{i}^{k+1}=\left\{x: \psi_{i}^{k+1}(x)>0\right\} .
$$

The behavior of (5.8) on $\mathcal{J}_{2}$, i.e., along one of the smooth surfaces $\Gamma_{i, j}$ away from any junctions, can be understood by simply Taylor-expanding the convolutions. To that end, take a $p \in \Gamma_{i, j} \backslash \mathcal{J}_{\geq 3}$. Near $p$, we have

$$
G_{\delta t} * u_{i}^{k} \approx G_{\delta t} * u_{j}^{k} \approx \frac{1}{2}
$$

while $G_{\delta t} * u_{\ell}^{k}$ for $\ell \notin\{i, j\}$ is exponentially small in $\delta t$ near $p$. Thus, near $p$, the coefficients $\phi_{\ell}^{k}(x)$ given by (5.7) become

$$
\phi_{\ell}^{k} \approx \begin{cases}\sigma_{\ell, i} G_{\delta t} * u_{i}^{k}+\sigma_{\ell, j} G_{\delta t} * u_{j}^{k} & \text { if } \ell \notin\{i, j\}, \\ \sigma_{i, j} G_{\delta t} * u_{j}^{k} & \text { if } \ell=i, \\ \sigma_{i, j} G_{\delta t} * u_{i}^{k} & \text { if } \ell=j,\end{cases}
$$

with an error that is exponentially small in $\delta t$. If the surface tensions $\sigma_{i, j}$ satisfy the strict triangle inequality, this implies

$$
\min \left\{\phi_{i}^{k}(x), \phi_{j}^{k}(x)\right\}<\phi_{\ell}^{k}(x) \text { for all } \ell \notin\{i, j\}
$$

for $x$ near $p$. Hence, wetting does not occur: no new phase gets nucleated along $\Gamma_{i, j}$. The updated interface $\Gamma_{i, j}$ can then be located by the equation

$$
\sigma_{i, j} G_{\delta t} * u_{j}^{k}(x) \approx \sigma_{i, j} G_{\delta t} * u_{i}^{k}(x)
$$

in a neighborhood of $p$, where once again the error is exponentially small in $\delta t$.

Let $n_{i, j}(p)$ denote the unit normal to $\Gamma_{i, j}$ at $p$, pointing from $\Sigma_{i}$ into $\Sigma_{j}$; see Figure 5.1. Taking the kernel $G_{\delta t}$ to be the Gaussian (3.1) and Taylor-expanding 


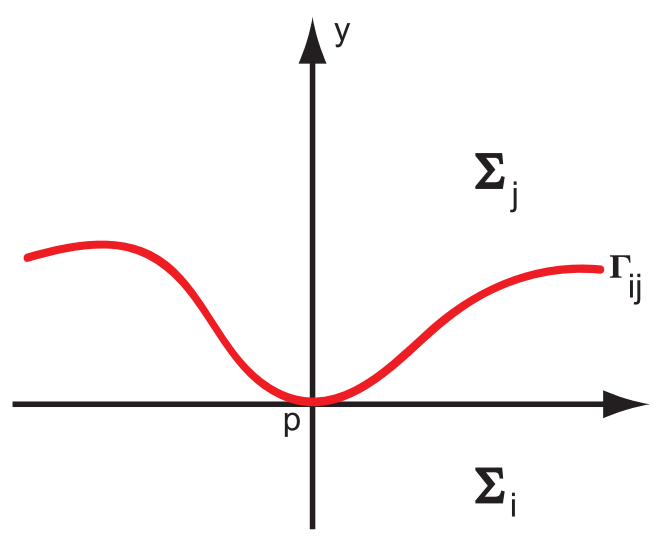

FiguRE 5.1. The behavior of the algorithm at a point $p$ on the smooth interface $\Gamma_{i, j}$ between two phases $\Sigma_{i}$ and $\Sigma_{j}$.

the convolutions as in, e.g., [34], we find

$$
G_{\delta t} * u_{i}^{k}\left(p+y n_{i, j}(p)\right)=\frac{1}{2}-\frac{1}{2 \sqrt{\pi} \sqrt{\delta t}} y+\frac{\sqrt{\delta t}}{2 \sqrt{\pi}} \kappa_{i, j}^{k}(p)+O(\delta t) .
$$

Using $u_{j}^{k}=1-u_{i}^{k}$ near $p$, this expansion in (5.12) yields

$$
y \approx \kappa_{i, j}^{k}(p) \delta t,
$$

which implies that the point $p$ on the interface $\Gamma_{i, j}$ moves by the normal speed

$$
v_{\perp}(p) \approx \kappa_{i, j}^{k}(p)
$$

at the end of update (5.6). In other words, the mobility of interface $\Gamma_{i, j}$ under update rule 5.6 is given by

$$
\mu_{i, j}=\frac{1}{\sigma_{i, j}} .
$$

Remark. If on the other hand the surface tensions $\sigma_{i, j}$ are positive but fail to satisfy the triangle inequality, the foregoing discussion is invalid. In fact, numerical experiments show that in those cases update (5.8), as well as its modifications discussed below, can lead to wetting by instantaneously nucleating a new phase along one of the existing interfaces. If desired, nucleation can be easily disallowed by restricting the optimization (5.5) at a given point $x \in D$ to only those phases that are already present in a neighborhood of $x$. All essential properties of the algorithm discussed in subsequent sections remains intact under this modification.

\section{Algorithm for General Mobilities}

To advance the interfaces with more general mobilities, we bring in retardation terms, to be added to the energy. One (computationally expensive and thus ultimately undesirable) approach to designing retardation terms is to use (much as 
in [2] and [24]) the signed distance function of the grains at the $k^{\text {th }}$ time step to limit their movement to the $(k+1)^{\text {th }}$ time step. To that end, let $d_{i}^{k}(x)$ denote the signed distance function of $\Sigma_{i}^{k}$ :

$$
d_{i}^{k}(x)= \begin{cases}\min _{y \in\left(\Sigma_{i}^{k}\right)^{\mathrm{c}}|x-y|} & \text { if } x \in \Sigma_{i}^{k}, \\ -\min _{y \in \Sigma_{i}^{k}}|x-y| & \text { if } x \in\left(\Sigma_{i}^{k}\right)^{\mathrm{c}} .\end{cases}
$$

Consider the retardation function

$$
\widetilde{R}_{i}^{k}(x):=\max _{j \neq i} \tilde{\gamma}_{i, j} d_{j}^{k}(x) .
$$

The (positive) constants $\tilde{\gamma}_{i, j}=\tilde{\gamma}_{j, i}$ will be specified subsequently. Regardless of their choice, $\widetilde{R}_{i}^{k}$ is a Lipschitz function that is nonpositive in $\Sigma_{i}^{k}$ and nonnegative elsewhere. More to the point, in a neighborhood of a point $p \in \Gamma_{i, j} \cap \mathcal{J}_{2}$, we have

$$
\tilde{R}_{\ell}^{k}(x)= \begin{cases}\tilde{\gamma}_{i, j} d_{i}^{k}(x)=-\tilde{\gamma}_{i, j} d_{j}^{k}(x) & \text { if } \ell=j, \\ \tilde{\gamma}_{i, j} d_{j}^{k}(x)=-\tilde{\gamma}_{i, j} d_{i}^{k}(x) & \text { if } \ell=i, \\ \max \left\{\tilde{\gamma}_{i, \ell} d_{i}^{k}(x), \tilde{\gamma}_{j, \ell} d_{j}^{k}\right\} & \text { if } \ell \notin\{i, j\},\end{cases}
$$

for all $x$ in that neighborhood. Consider modifying energy (4.5) at time step $k$ as follows:

$$
\widetilde{F}_{\delta t}^{k}\left(u_{1}, \ldots, u_{N}\right)=E_{\delta t}\left(u_{1}, \ldots, u_{N}\right)+\frac{2}{\delta t} \sum_{i=1}^{N} \int u_{i} \widetilde{R}_{i}^{k} d x
$$

Note that since the additional terms are linear, convexity properties of (5.16) are the same as that of 4.5). Moreover, we have

$$
\widetilde{R}_{i}^{k}(x) \begin{cases}\leq 0 & \text { if } x \in \Sigma_{i}^{k}, \\ \geq 0 & \text { otherwise, }\end{cases}
$$

and therefore it easily follows from $(5.16)$ that

$$
\widetilde{F}_{\delta t}^{k}(u) \leq \widetilde{F}_{\delta t}\left(u^{k}\right) \Rightarrow E_{\delta t}(u) \leq E_{\delta t}\left(u^{k}\right) .
$$

The linearization of this new energy at $u^{k}$ is

$$
\mathcal{L}_{F_{\delta t}}\left(u^{k}, u\right)=\mathcal{L}_{E_{\delta t}}\left(u^{k}, u\right)+\frac{2}{\delta t} \sum_{i} \int u_{i} \widetilde{R}_{i}^{k} d x
$$

up to terms constant in $u$. Minimization of the linear energy (5.17) over $\mathcal{K}$ leads to the modified thresholding scheme:

$$
u_{i}^{k+1}(x)= \begin{cases}1 & \text { if } \phi_{i}^{k}(x)+\frac{1}{\sqrt{\delta t}} \widetilde{R}_{i}^{k}(x)=\min _{\ell}\left(\phi_{\ell}^{k}(x)+\frac{1}{\sqrt{\delta t}} \widetilde{R}_{\ell}^{k}(x)\right) \\ 0 & \text { otherwise. }\end{cases}
$$

The corresponding level set function delineating the boundary of $\Sigma_{i}^{k+1}$ is given by

$$
\psi_{i}^{k+1}=\left(\min _{\ell \neq i}\left(\phi_{\ell}^{k}+\frac{1}{\sqrt{\delta t}} \widetilde{R}_{\ell}^{k}\right)\right)-\left(\phi_{i}^{k}+\frac{1}{\sqrt{\delta t}} \widetilde{R}_{i}^{k}\right) .
$$


As in the previous section, take a point $p \in \Gamma_{i, j} \cap \mathcal{J}_{2}$. Note that 5.15 implies $\widetilde{R}_{\ell}^{k}$ vanishes at $\Gamma_{i, j}$ for all $i, j, \ell$. That, together with (5.11), imply that in a neighborhood of $p$ we have

$$
\phi_{j}^{k}+\frac{1}{\sqrt{\delta t}} \widetilde{R}_{j}^{k}=\min _{\ell \neq i}\left(\phi_{\ell}^{k}+\frac{1}{\sqrt{\delta t}} \widetilde{R}_{\ell}^{k}\right)
$$

provided that the $\sigma_{i, j}$ satisfy the strict triangle inequality. Therefore,

$$
\begin{aligned}
\psi_{i}^{k+1} & =\phi_{j}^{k}+\frac{1}{\sqrt{\delta t}} \widetilde{R}_{j}^{k}-\left(\phi_{i}^{k}+\frac{1}{\sqrt{\delta t}} \widetilde{R}_{i}^{k}\right) \\
& \stackrel{5.15}{-} \phi_{j}^{k}+\frac{1}{\sqrt{\delta t}} \widetilde{\gamma}_{i, j} d_{i}^{k}-\phi_{i}^{k}-\frac{1}{\sqrt{\delta t}} \tilde{\gamma}_{i, j} d_{j}^{k} \\
& \stackrel{5.11}{\approx} 2\left(\sigma_{i, j}\left(G_{\delta t} * u_{i}^{k}-\frac{1}{2}\right)+\frac{1}{\sqrt{\delta t}} \tilde{\gamma}_{i, j} d_{i}^{k}\right) .
\end{aligned}
$$

Specialize $G_{\delta t}$ once again to the Gaussian kernel (3.1). Taylor-expanding the convolution in (5.20) as in (5.13) and observing that

$$
d_{j}^{k}(x)=-d_{i}^{k}(x)=(x-p) \cdot n_{i, j}(p)+O(\delta t) \text { for }|x-p|=O(\sqrt{\delta t})
$$

gives

$\psi_{i}^{k+1}\left(p+y n_{i, j}(p)\right)=-\frac{1}{\sqrt{\delta t}}\left(\frac{1}{\sqrt{\pi}} \sigma_{i, j}+2 \tilde{\gamma}_{i, j}\right) y+\frac{\sigma_{i, j}}{\sqrt{\pi}} \kappa_{i, j}(p) \sqrt{\delta t}+O(\delta t)$

for $y=O(\sqrt{\delta t})$. Solving for $y$, we see that the normal speed of the interface at $p$ is given by

Hence, choosing

$$
v_{\perp}(p)=\frac{\sigma_{i, j}}{2 \sqrt{\pi} \tilde{\gamma}_{i, j}+\sigma_{i, j}} \kappa_{i, j}(p) .
$$

$$
\tilde{\gamma}_{i, j}=\frac{1}{2 \sqrt{\pi}}\left(\frac{1}{\mu_{i, j}}-\sigma_{i, j}\right)
$$

leads to the desired normal speed

$$
v_{\perp}(p)=\mu_{i, j} \sigma_{i, j} \kappa_{i, j}(p)
$$

with the proviso that $\mu_{i, j}<1 / \sigma_{i, j}$, a condition easily accommodated via rescaling the variable $t$ as $t \rightarrow \alpha t$, if necessary.

As already mentioned, retardation terms (5.14) require computing the signed distance functions to the boundary of the grains at every time step. This can be readily accomplished by any one of the efficient redistancing algorithms developed in the level set literature. Nevertheless, computation of distance functions in a purely thresholding-based scheme is costly (compared to the other steps) from a practical and unappealing from an aesthetic point of view. It also turns out to be unnecessary: the convolutions computed at every time step can be used to construct proxies for the distance functions $d_{i}^{k}$. Indeed, the level set function $\sqrt{\delta t} \psi_{i}^{k}$ ought to serve as a good proxy for $d_{i}^{k}$ near $\Gamma_{i, j}$, up to a multiplicative factor independent 
of $\delta t$. To that end, consider replacing the retardation terms $\widetilde{R}_{i}^{k}$ in energy 5.16 with ones of the form

$$
R_{i}^{k}(x):=\max _{j \neq i} \gamma_{i, j} \sqrt{\delta t} \psi_{j}^{k}(x),
$$

where $\gamma_{i, j}=\gamma_{j, i}$ once again denote positive constants that will be subsequently specified. For $x$ near $p$, update (5.19) then becomes

$$
\psi_{i}^{k+1}=\left(\phi_{j}^{k}+\gamma_{i, j} \psi_{j}^{k}\right)-\left(\phi_{i}^{k}+\gamma_{i, j} \psi_{i}^{k}\right) .
$$

We now hypothesize that the $\psi_{i}^{k}$ generated by update (5.24) will satisfy

$$
\psi_{i}^{k}=C_{i, j}^{k} \frac{1}{\sqrt{\delta t}} d_{i}^{k}+O(\delta t)
$$

for all $x$ such that $|x-p|=o(\sqrt{\delta t})$; we will concurrently verify this form and determine the $C_{i, j}^{k}$ with an informal calculation. Under this hypothesis, (5.24) becomes

$$
\psi_{i}^{k+1}=2\left(\sigma_{i, j}\left(G_{\delta t} * u_{i}^{k}-\frac{1}{2}\right)+C_{i, j}^{k} \frac{1}{\sqrt{\delta t}} \gamma_{i, j} d_{i}^{k}\right)+O(\delta t) .
$$

Utilizing the Taylor expansions (5.13) and (5.21) once again, we see

$$
\begin{aligned}
\psi_{i}^{k+1}\left(p+y n_{i, j}(p)\right)= & -\frac{1}{\sqrt{\delta t}}\left(\frac{\sigma_{i, j}}{\sqrt{\pi}}+2 \gamma_{i, j} C_{i, j}^{k}\right) y \\
& +\frac{\sqrt{\delta t}}{\sqrt{\pi}} \sigma_{i, j} \kappa_{i, j}(p)+O(\delta t) .
\end{aligned}
$$

Solving for $y$, we see that the interface moves by normal speed

$$
v_{\perp}(p)=\frac{\sigma_{i, j}}{\sigma_{i, j}+2 \gamma_{i, j} \sqrt{\pi} C_{i, j}^{k}} \kappa_{i, j}(p)
$$

at the $k^{\text {th }}$ step of the algorithm, and the new level set function $\psi_{i}^{k+1}$ satisfies

$$
\psi_{i}^{k+1}=\frac{1}{\sqrt{\delta t}}\left(\frac{\sigma_{i, j}}{\sqrt{\pi}}+2 \gamma_{i, j} C_{i, j}^{k}\right) d_{i}^{k+1}+O(\delta t)
$$

in a neighborhood of its 0 -level set. This means the $C^{k}$ satisfy the simple recurrence

with the fixed point

$$
C_{i, j}^{k+1}=\frac{\sigma_{i, j}}{\sqrt{\pi}}+2 \gamma_{i, j} C_{i, j}^{k}
$$

$$
C_{i, j}=\frac{\sigma_{i, j}}{\sqrt{\pi}\left(1-2 \gamma_{i, j}\right)}
$$

that is globally asymptotically stable as long as $\gamma_{i, j} \in\left(0, \frac{1}{2}\right)$. Thus, as long as $0<\sigma_{i, j} \mu_{i, j}<1$, we may choose

$$
\gamma_{i, j}=\frac{1}{2}\left(1-\mu_{i, j} \sigma_{i, j}\right)
$$


and by (5.25) obtain exponential (in time step $k$ ) convergence to the desired normal speed (5.23).

Putting it all together, and in summary, the proposed algorithm for general mobilities $\mu_{i, j}$, corresponding to the normal interfacial speed

$$
v_{\perp}(p)=\mu_{i, j} \sigma_{i, j} \kappa_{i, j}(p)
$$

along $\Gamma_{i, j}$ and subject to Herring angle conditions (1.7), is as follows:

Algorithm: Given the initial partition $\Sigma_{1}^{0}, \ldots, \Sigma_{N}^{0}$ with
$\Sigma_{i}^{0}=\left\{x: \psi_{i}^{0}(x)>0\right\}$, to obtain the partition $\Sigma_{1}^{k+1}, \ldots, \Sigma_{N}^{k+1}$ at time
step $t=(\delta t)(k+1)$ from the partition $\Sigma_{1}^{k}, \ldots, \Sigma_{N}^{k}$ at time $t=(\delta t) k$ :

1. Form the convolutions:

$$
\phi_{i}^{k}=G_{\delta t} *\left(\sum_{j=1}^{N} \sigma_{i, j} \mathbb{1}_{\Sigma_{j}^{k}}\right) \text { for } i=1,2, \ldots N,
$$

where $G_{\delta t}$ is the Gaussian (3.1).

2. Form the retardation functions:

$$
R_{i}^{k}=\max _{j \neq i} \frac{\sqrt{\delta t}}{2}\left(1-\mu_{i, j} \sigma_{i, j}\right) \psi_{j}^{k} .
$$

3. Form the comparison functions:

$$
\psi_{i}^{k+1}=\left(\min _{\ell \neq i} \phi_{\ell}^{k}+\frac{1}{\sqrt{\delta t}} R_{\ell}^{k}\right)-\left(\phi_{i}^{k}+\frac{1}{\sqrt{\delta t}} R_{i}^{k}\right) .
$$

4. Threshold the comparison functions $\psi_{i}^{k+1}$ :

$$
\Sigma_{i}^{k+1}=\left\{x: \psi_{i}^{k+1}(x)>0\right\} .
$$

If we define the energy

$$
F_{\delta t}^{k}\left(u_{1}, \ldots, u_{N}\right)=E_{\delta t}\left(u_{1}, \ldots, u_{N}\right)+\frac{2}{\delta t} \sum_{i=1}^{N} \int u_{i} R_{i}^{k} d x
$$

we see that the algorithm above consists of the optimization

$$
u_{k+1}=\underset{\mathrm{u} \in \mathcal{K}}{\arg -\min } \mathcal{L}_{F_{\delta t}^{k}}\left(u^{k}, u\right)
$$

where $\mathcal{L}_{F_{\delta t}^{k}}\left(u^{k}, \cdot\right)$ denotes the linearization of $F_{\delta t}^{k}$ at $u^{k}$. And in fact, it can be easily shown that

$$
F_{\delta t}^{k}(u) \leq F_{\delta t}^{k}\left(u^{k}\right) \Rightarrow E_{\delta t}(u) \leq E_{\delta t}\left(u^{k}\right)
$$


see the proof of Proposition 5.3 below in Section 5.2. In other words, the presence of the time-step-dependent retardation terms $R_{i}^{k}$ in (5.32) — which were introduced to fix up mobilities without modifying angle conditions-does not influence whether the original interfacial energy $E_{\delta t}$ in (4.5) is dissipated or not by an algorithm, such (5.26)-(5.29), that implements (5.31).

\subsection{Stability of the Algorithm}

In this section, we investigate conditions under which Algorithm 5.26)-(5.29) introduced in Section 5.1 turns out to be unconditionally gradient stable: under certain assumptions on the surface tension matrix $\sigma$, it dissipates energy (5.30) and thus (4.2) at every iteration, regardless of the width of the convolution kernel $G$ (e.g., the time step size $\delta t$ appearing in the Gaussian (3.1) used in Step (5.26) of the algorithm. Although the convolution kernel $G$ is typically taken to be the Gaussian 3.1, in this section we merely require it to satisfy the following two conditions: $G(x) \geq 0$ for all $x$ and

$$
\widehat{G}(\xi) \geq 0 \text { for all } \xi
$$

where $\widehat{G}$ denotes the Fourier transform of $G$. By virtue of $(5.33)$, we can define a new kernel $g$ such that

$$
G=g * g .
$$

First note the following simple general fact:

LEMma 5.2. Let $\mathbb{X}$ be a Hilbert space. Let $\mathcal{K} \subset \mathbb{X}$ be a closed, bounded, convex set. Let $F: \mathbb{X} \rightarrow \mathbb{R}$ be Fréchet differentiable and concave on $\mathcal{K}$. Consider the following minimization scheme:

$$
x_{*} \in \underset{\mathrm{x} \in \mathcal{K}}{\arg -\min } \mathcal{L}_{F}\left(x_{0}, x\right)
$$

where $\mathcal{L}_{F}\left(x_{0}, \cdot\right)$ denotes the linearization of $F$ at $x_{0} \in \mathcal{K}$. Then:

$$
F\left(x_{*}\right) \leq F\left(x_{0}\right) .
$$

ProOF. By concavity of $F$, we have

$$
F\left(x_{*}\right) \leq \mathcal{L}_{F}\left(x_{0}, x_{*}\right) .
$$

By optimality of $x_{*}$ for $\mathcal{L}_{F}\left(x_{0}, \cdot\right)$ on $\mathcal{K}$

$$
\mathcal{L}_{F}\left(x_{0}, x_{*}\right) \leq \mathcal{L}_{F}\left(x_{0}, x_{0}\right)=F\left(x_{0}\right) .
$$

Combining the two inequalities above leads to the desired conclusion.

We will consider surface tension matrices $\sigma$ that are conditionally negative semidefinite:

$$
\left\{\sigma \in \mathcal{S}^{N}: \sum_{i, j=1}^{N} \sigma_{i, j} \xi_{i} \xi_{j} \leq 0 \text { whenever } \sum_{i=1}^{N} \xi_{i}=0\right\}
$$

In words, these matrices are negative semidefinite as quadratic forms on $(1, \ldots$, $1)^{\perp}$. Lemma 5.2 implies the following: 
Proposition 5.3. Let the surface tensions matrix $\sigma \in \mathcal{S}_{N}$ be conditionally negative semidefinite. Then, Algorithm (5.26)-(5.29) is unconditionally gradient stable: each time step dissipates the energy $E_{\delta t}$ on partitions given in (4.2), its relaxation given in (4.5), and its modified relaxation $F_{\delta t}$ given in (5.30).

PROof. Consider the step $k$ of Algorithm [5.26)-(5.29). The relaxed energy $F_{\delta t}^{k}$ at time step $k$, given in 5.30, is quadratic, and is thus easily seen to be Fréchet differentiable on $\left(L^{2}(D)\right)^{N}$. The constraint set $\mathcal{K}$ given in (5.1) is a closed, bounded, convex subset of $\left(L^{2}(D)\right)^{N}$. Take a point $u^{0} \in \mathcal{K}$; we have

$$
\begin{aligned}
F_{\delta t}^{k}(u)= & F_{\delta t}^{k}\left(u-u^{0}\right)+\text { linear terms in } u \\
= & \sum_{i, j} \int \sigma_{i, j}\left(u-u^{0}\right)_{i} G *\left(u-u^{0}\right)_{j} d x+\text { linear terms in } u \\
= & \sum_{i, j} \int \sigma_{i, j}\left(g *\left(u-u^{0}\right)\right)_{i}\left(g *\left(u-u^{0}\right)\right)_{j} d x \\
& + \text { linear terms in } u .
\end{aligned}
$$

Since $\sigma$ is conditionally negative semidefinite by hypothesis, and $\sum_{i}\left(u-u^{0}\right)_{i}=0$ for $u \in \mathcal{K}$, we see that $F_{\delta t}^{k}$ is concave on $\mathcal{K}$. Therefore, Lemma 5.2 applies to $F_{\delta t}^{k}$ on $\mathcal{K}$. The linearization of $F_{\delta t}^{k}$ at $\mathbb{1}_{\Sigma^{k}}$ is

$$
\mathcal{L}_{F_{\delta t}^{k}}\left(\mathbb{1}_{\Sigma^{k}}, u\right)=\frac{2}{\sqrt{\delta t}} \int \sum_{i=1}^{N} u_{i}\left(\phi_{i}^{k}+R_{i}^{k}\right) d x
$$

up to terms constant in $u$; here the $\phi_{i}^{k}$ are as in step 5.26) of the algorithm, and $R_{i}^{k}$ are as in step (5.27). The minimizer of $\mathcal{L}_{F_{\delta t}^{k}}$ on $\mathcal{K}$ is given by $\mathbb{1}_{\Sigma^{k+1}} \in \mathcal{B}$ where $\Sigma^{k+1}$ is as defined in step 5.29) of the algorithm, since 5.36 can be minimized over $\mathcal{K}$ by minimizing its integrand at each point $x \in D$ independently, leading to (5.29). This establishes that Algorithm (5.26)-5.29) dissipates energy (5.30) at every time step.

Next, note that by (5.27) and (5.29) the retardation terms satisfy

$$
R_{i}^{k}(x) \begin{cases}\geq 0 & \text { if } x \in\left(\Sigma_{i}^{k}\right)^{\mathrm{c}}, \\ \leq 0 & \text { if } x \in \Sigma_{i}^{k}\end{cases}
$$

Therefore,

$$
\sum_{i=1}^{N} \int \mathbb{1}_{\Sigma_{i}^{k}} R_{i}^{k} d x \leq \sum_{i=1}^{N} \int u_{i} R_{i}^{k} d x
$$

for any $u \in \mathcal{B}$. Since $F_{\delta t}^{k}\left(\Sigma^{k+1}\right) \leq F_{\delta t}^{k}\left(\Sigma^{k}\right)$, this means

$$
E_{\delta t}\left(\mathbb{1}_{\Sigma^{k+1}}\right) \leq E_{\delta t}\left(\mathbb{1}_{\Sigma^{k}}\right) .
$$


Thus, Algorithm (5.26)-(5.29) dissipates energy (4.5), and equivalently (4.2) at every time step.

We now explore conditions under which the matrix of surface tensions $\sigma_{i, j}$ is conditionally negative semidefinite, so that Proposition 5.3 applies. These matrices turn out to be extensively studied in various contexts, so we quote a number of standard results. Here is an outline of the discussion to follow:

- The triangle inequality by itself is neither necessary nor sufficient to guarantee that a $\sigma \in \mathcal{S}_{N}$ is conditionally negative semidefinite. However, a necessary condition turns out to be that the matrix $\sqrt{\sigma_{i, j}}$ satisfy the triangle inequality. An example of a conditionally negative semidefinite $\sigma$ violating the triangle inequality (i.e., $\sigma \notin \mathcal{T}_{N}$ ) is discussed in Section 6 .

- Conditional negative semidefiniteness of an $N \times N$ matrix $\sigma$ turns out to be connected to embeddability of finite metric spaces: According to [37], a matrix $\sigma_{i, j}$ is conditionally negative semidefinite if and only if there exist points $p_{1}, \ldots, p_{N} \in \mathbb{R}^{M}$ for some $M$ such that $\sigma_{i, j}=\left|p_{i}-p_{j}\right|_{2}^{2}$. In words, the matrix $\sigma$ should arise as the matrix of squared distances for a finite metric space embeddable into the euclidean space $\ell^{2}\left(\mathbb{R}^{M}\right)$ for some $M$.

In the same vein, it turns out that if $\sigma_{i, j}=\left|p_{i}-p_{j}\right|_{1}$ for some points $p_{1}, \ldots, p_{N} \in \mathbb{R}^{M}$ for some $M$, then $\sigma$ is conditionally negative semidefinite. In words, a sufficient condition is that $\sigma$ arise as the matrix of distances for a finite metric space that is embeddable into $\ell^{1}\left(\mathbb{R}^{M}\right)$ for some $M$. This is a large subset of $\mathcal{T}_{N}$ and has a neat interpretation from the point of view of numerical methods.

- A well-known model of how surface tensions arise due to dislocations in a simple cubic lattice was proposed by Read and Shockley in [32]. There the surface tension of the interface between two neighboring grains is shown to have a functional dependence of a certain form on the misorientation between the two grains on either side. It turns out that when the crystallographic orientations of the grains differ from each other by rotations about a fixed axis (sometimes called the fiber-texture, or the two-dimensional crystallography setting), all surface tensions models broadly resembling that of Read and Shockley, when taken together with a high-angle saturation assumption [19], are conditionally negative semidefinite. This is shown in Theorem 5.5 .

More realistically (i.e., in the so-called three-dimensional crystallography case), when the orientations of the grains in the network differ from each other by arbitrary rotations about an arbitrary axis, we can use the very specific functional dependence (see formula (5.44)) of surface tension on the misorientation angle that is widely accepted and used in materials science literature to still show that the resulting surface tension matrix is conditionally negative semidefinite. This is shown in Theorem 5.6. Thus, 
algorithms (5.7)-(5.8) and the more general (5.26) $-(5.29)$ proposed in this paper are unconditionally gradient stable for the important class of Read and Shockley grain models.

- There are many studies in materials science that describe deviations from Read-Shockley-type surface tensions, e.g., [7]. It is therefore of interest to at least find a variant of Algorithm (5.26)-(5.29) that can be guaranteed to dissipate energy (4.2) for as wide a class of surface tensions as possible. It turns out that a Gauss-Seidel version of (5.26)-(5.29) can be devised that is guaranteed to dissipate (4.2) for all triangle-inequality-satisfying surface tensions. This algorithm is described and its properties established in Proposition 5.7 .

We now explain these points in detail. The terminology and basic facts relating to embeddability of finite metric spaces are taken from [4].

Any triangle-inequality-satisfying set of surface tensions $\sigma_{i, j}$ defines a metric on the finite set of $N$ elements $\left\{p_{1}, \ldots, p_{N}\right\}$ :

$$
d\left(p_{i}, p_{j}\right)=\sigma_{i, j}
$$

Given a set of $N$ points, a metric $d$ on this set of points is called a cut metric if it has the following form:

$$
d_{S}\left(p_{i}, p_{j}\right)= \begin{cases}0 & \text { if } i \in S \text { and } j \in S \\ 0 & \text { if } i \in S^{\mathrm{c}} \text { and } j \in S^{\mathrm{c}} \\ 1 & \text { otherwise }\end{cases}
$$

for some $S \subseteq\{1,2, \ldots, N\}$. We write $d_{S}$ to denote the cut metric associated with the set of indices $S$. The following turns out to be a standard fact [8, prop. 4.2.2]:

Fact 1. A metric $d$ on the set of $N$ points $\left\{p_{1}, \ldots, p_{N}\right\}$ can be embedded into $\ell^{1}\left(\mathbb{R}^{M}\right)$ for some $M$ if and only if $d$ is a positive sum of cut metrics:

$$
d\left(p_{i}, p_{j}\right)=\sum_{k} \alpha_{k} d_{S_{k}}\left(p_{i}, p_{j}\right)
$$

where $\alpha_{k} \geq 0$ and $S_{k} \subseteq\{1,2, \ldots, N\}$.

The next claim follows easily from this and gives another partial characterization of surface tensions that Proposition 5.3 applies to.

COROLlary 5.4. Let $\sigma \in \mathcal{T}_{N}$. If $\sigma_{i, j}$ can be embedded into $\ell^{1}\left(\mathbb{R}^{M}\right)$ for some $M$ when viewed as pairwise distances on a finite set of $N$ elements, then $\sigma$ defines a negative form on $(1,1, \ldots, 1)^{\perp} \subset \mathbb{R}^{N}$. Therefore, Algorithm (5.26)-(5.29) is unconditionally gradient stable for such a choice of surface tensions: it dissipates energies (4.2), (4.5), and (5.16). 
ProOF. By Fact 1 , it is sufficient to establish negativity of $\sigma \in \mathcal{T}_{N}$ of the form

$$
\sigma_{i, j}= \begin{cases}0 & \text { if } i \in S \text { and } j \in S, \\ 0 & \text { if } i \in S^{\mathrm{c}} \text { and } j \in S^{\mathrm{c}} \\ 1 & \text { otherwise, }\end{cases}
$$

where $S \in\{1,2, \ldots, N\}$. Let $\xi \in(1,1, \ldots, 1)^{\perp} \subset \mathbb{R}^{N}$. We have

$$
\sum_{i \in S^{\mathrm{c}}} \xi_{i}=-\sum_{i \in S} \xi_{i}
$$

Therefore,

$$
\sum_{i, j} \sigma_{i, j} \xi_{i} \xi_{j}=2 \sum_{\substack{i \in S^{c} \\ j \in S^{\mathrm{c}}}} \xi_{i} \xi_{j}=2 \sum_{i \in S} \xi_{i} \sum_{j \in S^{\mathrm{c}}} \xi_{j}=2\left(\sum_{i \in S} \xi_{i}\right)\left(-\sum_{i \in S} \xi_{i}\right) .
$$

The last expression above is concave in $\sum_{i \in S} \xi_{i}$ and therefore also in $\xi$.

Remark. Corollary 5.4 covers a large subset of triangle-inequality-satisfying surface tensions $\mathcal{T}_{N}$. A much smaller subset of this class are the additive surface tensions: these are of the form

$$
\sigma_{i, j}=\sigma_{i}+\sigma_{j} \quad \text { for } i \neq j
$$

where $\sigma_{i}$ are arbitrary positive constants. The resulting surface tension matrix $\sigma_{i, j}$ is easily seen to be conditionally negative definite, so additive surface tensions constitute a special case of the surface tensions covered by Corollary 5.4 The corresponding surface energies are the subset of energies of the form (1.2) that can be written as a positive sum of perimeters of the phases $\Sigma_{1}, \ldots, \Sigma_{N}$ :

$$
E=\sum_{i} \sigma_{i} \operatorname{Per}\left(\Sigma_{i}\right)
$$

Energy (1.2) cannot always be put in form (5.38), since the number of degrees of freedom in (5.38) is merely $N$ versus the $N$-choose- 2 degrees of freedom in $(1.2)$. When $N=3$, any triangle-inequality-satisfying set of surface tensions is additive. For $N \geq 4$, this is no longer the case, and the class of surface tensions covered by Corollary 5.4 is a much larger subset of all triangle-inequality-satisfying surface tensions than (5.37). Indeed, this larger class corresponds to surface energies (1.2) that can be written as

$$
E=\sum_{S \subseteq\{1,2, \ldots, N\}} \sigma_{S} \operatorname{Per}\left(\bigcup_{i \in S} \Sigma_{i}\right)
$$

where $S \subseteq\{1,2, \ldots, N\}$ and $\sigma_{S}$ are arbitrary positive constants. In words, these are energies that can be written as a positive sum of perimeters of arbitrary unions of the phases $\Sigma_{1}, \ldots, \Sigma_{N}$.

A number of earlier, well-known numerical algorithms for (1.2), such as [41], are restricted to the very small class of additive energies (5.38). The interesting recent approach of [35,36] also appears to be restricted to this very special case. 




FIGURE 5.2. $\ell^{1}$-embeddable metrics constitute a strict subset of the surface tensions that are covered by Proposition 5.3 .

Examples of non- $\ell^{1}$ embeddable metrics can be found in, e.g., [8]. They are good candidates for $\sigma \in \mathcal{S}_{N}$ that possibly might not be conditionally negative semidefinite. It turns out that when $N \leq 4$, all metrics are $\ell^{1}$ embeddable (and thus such a $\sigma_{i, j}$ is conditionally negative semidefinite); see, e.g., [4]. So the simplest example of a grain boundary model for which Proposition 5.3 potentially does not apply contains five phases. The fact that any $\sigma_{i, j} \in \mathcal{T}_{N}$ with $N \leq 4$ is conditionally negative semidefinite appears previously also in [17].

Example. Consider the matrix

$$
\sigma^{*}(a, b)=\left(\begin{array}{ccc|cc}
0 & b & b & a & a \\
b & 0 & b & a & a \\
b & b & 0 & a & a \\
\hline a & a & a & 0 & b \\
a & a & a & b & 0
\end{array}\right) .
$$

In order for $\sigma_{i, j}^{*}(a, b)$ to satisfy the triangle inequality, we must have

$$
a, b \geq 0 \quad \text { and } \max \{a, b\} \leq 2 \min \{a, b\} .
$$

If $b \in\left(\frac{3}{2} a, 2 a\right)$, then $\sigma^{*}(a, b)$ is non- $\ell^{1}$ embeddable, as it then fails to satisfy a pentagon inequality; see, e.g., [4]. It turns out that this is not sufficient for $\sigma^{*}(a, b)$ to violate negativity on $(1,1, \ldots, 1)^{\perp}$. Indeed, we have, for example:

(1) $\sigma^{*}(1,1.6)$ is non- $\ell^{1}$ embeddable, but conditionally negative definite, and

(2) $\sigma^{*}(1,1.8)$ is not conditionally negative semidefinite.

Thus, $\sigma^{*}(1,1.8)$ is an example of triangle-inequality-satisfying set of surface tensions that falls outside the scope of Proposition 5.3. In fact, the approximate energy (4.5) is not quasi-concave (i.e., not all its superlevel sets are convex) on the constraint set $\mathcal{K}$ for this $\sigma$. A direction of positivity for the corresponding quadratic form is $(-2,-2,-2,3,3)$, which corresponds to phases 4 and 5 growing simultaneously at the expense of phases 1,2, and 3. The situation is summarized in Figure 5.2 . 
As the foregoing discussion shows, we are unable to establish that Algorithm (5.26)-5.29) dissipates energy (4.2) for all triangle-inequality-satisfying surface tensions; instead, we gave some partial results in this direction. Now, we add another special case to the class of surface tensions for which (5.26)-(5.29) can be shown to be dissipative: Read-Shockley surface tensions.

In two-dimensional crystallography, the orientation of a simple cubic lattice can be described by a single parameter: the angle $\theta$ of clockwise rotation about the origin that maps it back to the standard lattice $\mathbb{Z}^{2}$. Due to symmetries, one can take $\theta \in\left[-\frac{\pi}{4}, \frac{\pi}{4}\right]$ with the two ends of that interval identified. In the well-known work [32], Read and Shockley described a model for the grain boundary formed between two planar (or columnar in three dimensions) grains with cubic lattices. They obtained an expression for the surface tension (energy per unit area) of the grain boundary as a function of the misorientation angle between the two lattices under the proviso that the said angle is small. Together with a high-angle saturation assumption [19], the surface tension $\sigma_{i, j}$ of the interface between two grains with orientations $\overline{\theta_{i}}$ and $\theta_{j}$ has the form

$$
\sigma_{i, j}=\min _{k \in \mathbb{Z}} f\left(\left|\theta_{i}-\theta_{j}+k \frac{\pi}{2}\right|\right)
$$

where $f: \mathbb{R}^{+} \rightarrow \mathbb{R}$ satisfies

(1) $f \in C([0, \infty)) \cap C^{2}((0, \infty))$ and $\lim _{\xi \rightarrow 0^{+}} \xi^{2} f^{\prime}(\xi)=0$,

(2) $f(0)=0$ and $f(\xi) \geq 0$ for all $\xi$,

(3) $f^{\prime}(\xi) \geq 0$ for all $\xi>0$,

(4) $f^{\prime \prime}(\xi) \leq 0$ for all $\xi>0$.

See Figure 5.3 for an example.

THEOREM 5.5. Let the surface tensions $\sigma_{i, j}$ arise from the Read and Shockley law for two-dimensional crystallography. Then the $\sigma_{i, j}$ satisfy the triangle inequality. Moreover, as a quadratic form, $\sigma_{i, j}$ is conditionally negative semidefinite. Algorithm (5.26)-5.29) is thus unconditionally gradient stable for Read-and-Shockleytype grain boundary models for two-dimensional crystallography.

PROOF. First, let

$$
d_{i, j}=\min _{k \in \mathbb{Z}}\left|\theta_{i}-\theta_{j}+k \frac{\pi}{2}\right| .
$$

The $d_{i, j} \in[0, \pi / 4]$ are shortest distances among points on the circle $[-\pi / 2, \pi / 2]$, and therefore satisfy the triangle inequality. Take three distinct indices $i, j, k \in$ $\{1,2, \ldots, N\}$. With no loss of generality, assume that $d_{i, j} \geq d_{j, k}$. Then:

$$
f\left(d_{i, j}+d_{j, k}\right) \leq f\left(d_{i, j}\right)+f^{\prime}\left(d_{i, j}\right) d_{j, k}
$$

by the concavity of $f$. Also,

$$
f^{\prime}\left(d_{i, j}\right) d_{j, k} \leq f^{\prime}\left(d_{j, k}\right) d_{j, k}
$$




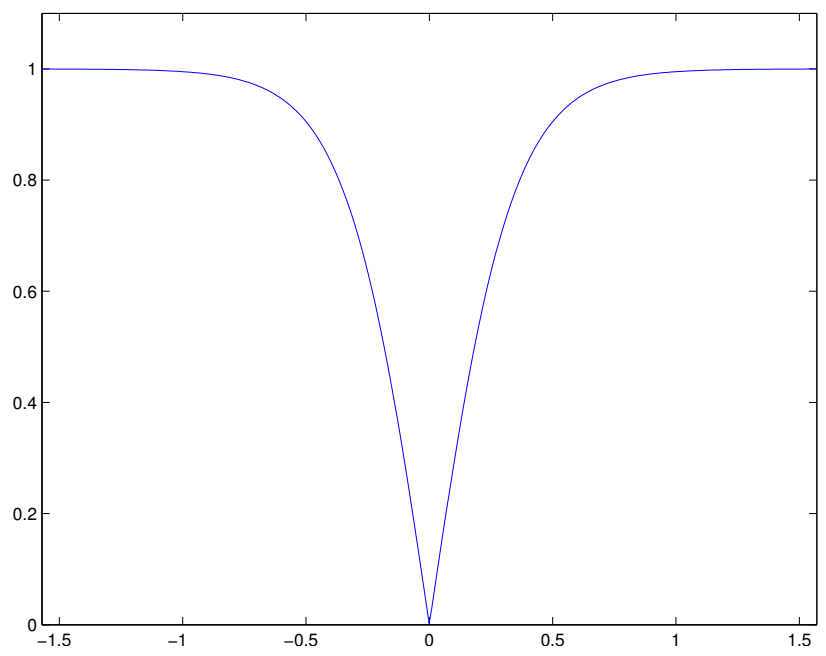

FIGURE 5.3. According to Read and Shockley [32], the typical dependence on the misorientation angle $\theta=\min _{k \in \mathbb{Z}}\left|\theta_{i}-\theta_{j}+k \pi / 2\right|$ of the surface tension $\sigma_{i, j}(\theta)$ associated with a grain boundary formed between two planar grains with cubic lattices.

and

$$
f\left(d_{j, k}\right)=\int_{0}^{d_{j, k}} f^{\prime}(\xi) d \xi \geq f^{\prime}\left(d_{j, k}\right) d_{j, k}
$$

Together, these mean

$$
f\left(d_{i, j}+d_{j, k}\right) \leq f\left(d_{i, j}\right)+f\left(d_{j, k}\right) .
$$

Since $f$ is increasing,

$$
f\left(d_{i, j}+d_{j, k}\right) \geq f\left(d_{i, k}\right)
$$

by the triangle inequality $d_{i, k} \leq d_{i, j}+d_{j, k}$. The last two inequalities now imply

$$
f\left(d_{i, k}\right) \leq f\left(d_{i, j}\right)+f\left(d_{j, k}\right),
$$

which establishes the triangle inequality for the $\sigma_{i, j}$.

Define the quadratic form

$$
Q(u)=\int_{-\pi / 4}^{\pi / 4} \int_{-\pi / 4}^{\pi / 4} f(x-y) u(x) u(y) d y d x
$$

where $f$ and $u$ are extended periodically to $\mathbb{R}$ with period $\frac{\pi}{2}$. Taking $u(\theta)=$ $\sum_{j} \xi_{j} \delta\left(\theta-\theta_{j}\right)$, we get

$$
Q(u)=\sum_{i, j} \sigma_{i, j} \xi_{i} \xi_{j} .
$$


Thus, it is sufficient to check that $Q(u) \leq 0$ for all $u$ with $\int u d \xi=0$. Expressing $Q(u)$ via the Fourier transform gives

$$
Q(u)=\sum_{n} \widehat{f}_{n}\left|\xi_{n}\right|^{2}
$$

Therefore, it is in fact sufficient to check that $\widehat{f}_{n} \leq 0$ for all $n \neq 0$. Moreover, since $f$ is even, it suffices to consider the cosine terms. We have

$$
\begin{aligned}
\widehat{f}_{n} & =2 \int_{-\pi / 4}^{\pi / 4} f(\xi) \cos (4 n \xi) d \xi=-\frac{1}{n} \lim _{\varepsilon \rightarrow 0^{+}} \int_{\varepsilon}^{\pi / 4} f^{\prime}(\xi) \sin (4 n \xi) d \xi \\
& =\frac{1}{4 n^{2}} \lim _{\varepsilon \rightarrow 0^{+}}\left((-1)^{n} f^{\prime}(\pi / 4)-f^{\prime}(\varepsilon) \cos (4 n \varepsilon)-\int_{\varepsilon}^{\pi / 4} f^{\prime \prime}(\xi) \cos (4 n \varepsilon) d \xi\right) \\
& \leq \frac{1}{4 n^{2}} \lim _{\varepsilon \rightarrow 0^{+}}\left((-1)^{n} f^{\prime}(\pi / 4)-f^{\prime}(\varepsilon) \cos (4 n \varepsilon)-\int_{\varepsilon}^{\pi / 4} f^{\prime \prime}(\xi) d \xi\right) \\
& =\frac{1}{4 n^{2}} \lim _{\varepsilon \rightarrow 0^{+}}\left(\left[(-1)^{n}-1\right] f^{\prime}(\pi / 4)+f^{\prime}(\varepsilon)(1-\cos (4 n \varepsilon))\right) \\
& =\frac{1}{4 n^{2}}\left[(-1)^{n}-1\right] f^{\prime}(\pi / 4) \leq 0 .
\end{aligned}
$$

where we integrated by parts twice and used the monotonicity, concavity, and behavior at 0 of $f$.

We now turn to the Read and Shockley model for three-dimensional crystallography, as described in [19]. A grain in three dimensions with a simple cubic crystal lattice can be described (nonuniquely) by a matrix $g \in \mathrm{SO}(3)$, i.e., by an orthogonal matrix with determinant +1 describing the rotation required to obtain the lattice of the grain from the standard integer lattice in $\mathbb{R}^{3}$. Any matrix $g \in \mathrm{SO}(3)$ can be described as a rotation by an angle $\theta \in[0, \pi]$ about an axis $v \in \mathbb{S}^{2}$. The rotation angle can be easily expressed as

$$
\theta(g)=\arccos \left(\frac{\operatorname{trace}(g)-1}{2}\right)
$$

whereas the axis $v$ is the eigenvector of $g$ corresponding to the eigenvalue 1 . The sign of $v$ is chosen so that the rotation angle is in the range $[0, \pi]$. When $\theta=\pi$, the two possible axes of rotation, namely $\pm v$, are identified with one another. $\mathrm{SO}(3)$ is thus equivalent to the unit ball in $\mathbb{R}^{3}$, the antipodal points on the surface of which have been identified.

The misorientation matrix between two grains with orientations $g_{i}$ and $g_{j}$ is then given by $g_{i} g_{j}^{-1}=g_{i} g_{j}^{\top}$. In [19], it is assumed that the surface tension $\sigma_{i, j}$ of the interface $\Gamma_{i, j}$ between the two grains depends only on the corresponding angle of the rotation $g_{i} g_{j}^{\top}$, not on the axis. In calculating the angle of rotation between $g_{i}$ and $g_{j}$, symmetries of the cubic lattice have to be taken into account. Let $\mathcal{O}$ denote the octahedral group (of symmetries of the cube in three dimensions), which has 
24 elements and is generated by 2 of them: right-handed $90^{\circ}$ rotations about any two of the three coordinate axes $\mathbf{i}, \mathbf{j}$, and $\mathbf{k}$. Note that

$$
\theta(r) \geq \frac{\pi}{2} \quad \text { if } r \in \mathcal{O} \text { and } r \neq \mathrm{Id} .
$$

Define the minimal angle of rotation $\theta_{\mathcal{O}}(g)$ of a $g \in \mathrm{SO}(3)$ as

$$
\theta_{\mathcal{O}}(g)=\min _{r \in \mathcal{O}} \theta(r g) \text {. }
$$

The misorientation angle between $g_{i}$ and $g_{j}$ is defined to be

$$
\theta_{i, j}=\theta_{\mathcal{O}}\left(g_{i} g_{j}^{\top}\right)
$$

and the corresponding surface tension $\sigma_{i, j}$ is given by

$$
\sigma_{i, j}=f\left(\theta_{i, j}\right)
$$

where $f$ is a function conforming to the properties listed previously in the twodimensional crystallography setting on page 832 . In the three-dimensional crystallography setting, we will in fact need to be more precise about the function $f$. As in [19, 32], we focus on the specific choice

$$
f(\theta)= \begin{cases}\frac{\theta}{\theta_{*}}\left(1-\log \left(\frac{\theta}{\theta_{*}}\right)\right) & \text { if } \theta<\theta_{*}, \\ 1 & \text { if } \theta \geq \theta_{*},\end{cases}
$$

where $\theta_{*}$ is a critical misorientation value. It denotes the rotation angle beyond which the surface tension saturates and, according to [19], has been experimentally determined to lie somewhere between $10^{\circ}$ and $30^{\circ}$.

THEOREM 5.6. Let the surface tension matrix $\sigma$ arise from the Read and Shockley law for three-dimensional crystallography, given according to (5.42) and (5.43) where $f$ satisfies conditions listed on page 832 Then, $\sigma$ satisfies the triangle inequality. Assume further that $f$ is given by the specific form (5.44) and that the critical misorientation angle $\theta_{*}$ in (5.44) satisfies $\theta_{*} \leq \frac{\pi}{4}=45^{\circ}$. Then, $\sigma$ is conditionally negative semidefinite. Therefore, Algorithm (5.26)-(5.29) is unconditionally gradient stable for the Read and Shockley grain boundary model for three-dimensional crystallography.

PROOF. Let us first establish the triangle inequality. For any three unit vectors $v_{1}, v_{2}$, and $v_{3}$ in $\mathbb{R}^{3}$, using the triangle inequality for the geodesic distance on $\mathbb{S}^{2}$ we have

$$
\arccos \left(v_{1} \cdot v_{3}\right) \leq \arccos \left(v_{1} \cdot v_{2}\right)+\arccos \left(v_{2} \cdot v_{3}\right) \text {. }
$$

Moreover, the angle of rotation $\theta(g) \in[0, \pi]$ of a rotation matrix $g$, given by formula (5.39), can be characterized as

$$
\theta(g)=\max _{\substack{v \in \mathbb{R}^{3} \\|v|=1}} \arccos (v \cdot g v)
$$


Combining these two, one gets

$$
\theta\left(g_{1} g_{2}\right) \leq \theta\left(g_{1}\right)+\theta\left(g_{2}\right) \text { for any } g_{1}, g_{2} \in \mathrm{SO}(3) .
$$

Given now $g_{i}, g_{j}$, and $g_{k}$ in $\mathrm{SO}(3)$, we have

$$
\begin{aligned}
& \theta_{i, k} \stackrel{5.42}{=} \min _{r \in \mathcal{O}} \theta\left(r g_{i} g_{k}^{\top}\right)=\min _{r_{1}, r_{2} \in \mathcal{O}} \theta\left(r_{2} r_{1}\left(g_{i} g_{j}^{\top}\right)\left(g_{j} g_{k}^{\top}\right)\right) \\
& \stackrel{5.39}{=} \min _{r_{1}, r_{2} \in \mathcal{O}} \theta\left(\left(r_{1} g_{i} g_{j}^{\top}\right)\left(g_{j} g_{k}^{\top} r_{2}\right)\right) \\
& \stackrel{5.47}{\leq} \min _{r_{1}, r_{2} \in \mathcal{O}}\left(\theta\left(r_{1} g_{i} g_{j}^{\top}\right)+\theta\left(g_{j} g_{k}^{\top} r_{2}\right)\right) \\
& \stackrel{5.39}{=} \theta_{i, j}+\theta_{j, k} .
\end{aligned}
$$

The triangle inequality for the corresponding surface tensions $\sigma_{i, j}=f\left(\theta_{i, j}\right)$ then follows as in the proof of Theorem 5.5 from the properties of the function $f$ listed on page 832

Turning to conditional negative semidefiniteness of $\sigma$, we will use a couple of rudimentary facts from the representation theory of $\mathrm{SO}(3)$; see, e.g., [9, 40]. The notation below follows chapter 14.4 of [9].

Define the function $h: \mathrm{SO}(3) \rightarrow \mathbb{R}$ as

$$
h(g)=f\left(\arccos \left(\frac{\operatorname{trace}(g)-1}{2}\right)\right)=f(\theta(g)) .
$$

We now argue that if $\theta_{*} \leq \frac{\pi}{4}$, we have

$$
\sum_{r_{1}, r_{2} \in \mathcal{O}} h\left(r_{1} g_{i}\left(r_{2} g_{j}\right)^{\top}\right)=24\left(\sigma_{i, j}+23\right)
$$

To see this, consider two cases:

Case $1 . \theta_{\mathcal{O}}\left(g_{i} g_{j}^{\top}\right) \leq \frac{\pi}{4}$.

By [5.41, this means there is $r_{*} \in \mathcal{O}$ such that $\theta\left(r_{*} g_{i} g_{j}^{\top}\right) \leq \frac{\pi}{4}$. If $r \in \mathcal{O}$ and $r \neq r_{*}$, then

$$
r_{*} r^{\top} \neq \mathrm{Id} \Rightarrow \theta\left(r_{*} r^{\top}\right) \geq \frac{\pi}{2}
$$

by (5.40), and by the triangle inequality (5.47)

$$
\begin{array}{r}
\theta\left(r r_{*}^{\top}\right) \leq \theta\left(r\left(g_{i} g_{j}^{\top}\right)\right)+\theta\left(\left(g_{i} g_{j}^{\top}\right)^{\top} r_{*}^{\top}\right) \\
\stackrel{5.39}{=} \theta\left(r\left(g_{i} g_{j}^{\top}\right)\right)+\theta\left(r_{*}\left(g_{i} g_{j}^{\top}\right)\right),
\end{array}
$$

which means

$$
\begin{aligned}
\theta\left(r g_{i} g_{j}^{\top}\right) & \geq \theta\left(r r_{*}^{\top}\right)-\theta\left(r_{*} g_{i} g_{j}^{\top}\right) \\
& \geq \frac{\pi}{2}-\frac{\pi}{4}=\frac{\pi}{4}
\end{aligned}
$$


By (5.41) and (5.42), this implies

$$
\theta_{i, j}=\theta\left(r_{*} g_{i} g_{j}^{\top}\right)
$$

and thus by 5.43,

$$
h\left(r_{*} g_{i} g_{j}^{\top}\right)=\sigma_{i, j}
$$

Therefore, and since $f(\theta)=1$ for $\theta \geq \frac{\pi}{4} \geq \theta_{*}$,

$$
h\left(r_{1} g_{i}\left(r_{2} g_{j}\right)^{\top}\right)=h\left(r_{2}^{\top} r_{1} g_{i} g_{j}^{\top}\right)= \begin{cases}\sigma_{i, j} & \text { if } r_{1}=r_{2} r_{*}, \\ 1 & \text { otherwise, }\end{cases}
$$

leading to 5.50 .

Case 2. $\theta_{\mathcal{O}}\left(g_{i} g_{j}^{\top}\right)>\frac{\pi}{4}$.

In this case $\theta\left(r g_{i} g_{j}^{\top}\right) \geq \frac{\pi}{4}$ for all $r \in \mathcal{O}$, so 5.50$)$ follows immediately from the definition 5.49) of $h$ and the fact that $f(\theta)=1$ for $\theta \geq \frac{\pi}{4}$.

Having established (5.50, next we define the following function $u$ on $\mathrm{SO}(3)$ :

$$
u(g)=\sum_{i} \xi_{i} \delta_{g_{i}}(g)
$$

where $\delta_{g}(\cdot)$ denotes the delta function centered at a point $g \in \mathrm{SO}(3)$. By (5.50), for any $\xi \in(1, \ldots, 1)^{\perp}$ we have

$$
\begin{aligned}
& \sum_{i, j} \sigma_{i, j} \xi_{i} \xi_{j} \\
& \stackrel{[5.50)}{-} \frac{1}{24} \sum_{r_{1}, r_{2} \in \mathcal{O}} \sum_{i, j} h\left(r_{1} g_{i}\left(r_{2} g_{j}\right)^{\top}\right) \xi_{i} \xi_{j} \\
& \stackrel{5.51]}{=} \frac{1}{24} \sum_{r_{1}, r_{2} \in \mathcal{O}_{\mathrm{SO}(3)}} \int_{\operatorname{SO}(3)} h\left(r_{1} g_{1}\left(r_{2} g_{2}\right)^{\top}\right) u\left(g_{1}\right) u\left(g_{2}\right) d g_{1} d g_{2} \\
& \quad=\frac{1}{24} \int_{\operatorname{SO}(3)} \int_{\operatorname{SO}(3)} h\left(g_{1} g_{2}^{\top}\right)\left(\sum_{r \in \mathcal{O}} u\left(r^{\top} g_{1}\right)\right)\left(\sum_{r \in \mathcal{O}} u\left(r^{\top} g_{2}\right)\right) d g_{1} d g_{2} .
\end{aligned}
$$

Here, $d g_{1}$ and $d g_{2}$ denote the left (as well as right) invariant measure of unit mass (i.e., the Haar measure) on $\mathrm{SO}(3)$. It is therefore sufficient to show that the quadratic form

$$
Q(u)=\int_{\operatorname{SO}(3) \operatorname{SO}(3)} \int_{1} h\left(g_{1} g_{2}^{\top}\right) u\left(g_{1}\right) u\left(g_{2}\right) d g_{1} d g_{2},
$$

defined on functions $u: \mathrm{SO}(3) \rightarrow \mathbb{R}$, is conditionally negative semidefinite. Let $\mathbf{0}^{m}$ denote the representation of $\mathrm{SO}(3)$ of weight $m$; this is an $(2 m+1) \times(2 m+1)$ 
unitary matrix-valued function on $\mathrm{SO}(3)$. The convolution defining the quadratic form $Q$ can be expressed as

$$
Q(u)=\sum_{m}(2 m+1) \operatorname{trace}\left(\widehat{u}^{m} \widehat{h}^{m}\left(\widehat{u}^{m}\right)^{*}\right)
$$

where ${ }^{\wedge}$ denotes the "Fourier transform," i.e.,

$$
\widehat{\phi}^{m}=\int_{\mathrm{SO}(3)} \phi(g)\left(\mathbf{o}^{m}\right)^{*}(g) d g
$$

for a function $\phi: \mathrm{SO}(3) \rightarrow \mathbb{R}$, with the inversion formula

$$
\phi(g)=\sum_{m}(2 m+1) \operatorname{trace}\left(\widehat{\phi}^{m} \mathbf{o}^{m}(g)\right) .
$$

See [9, p. 256].

Next, note that since $h$ depends only on the angle of rotation of a matrix $g$, it is a class function, and therefore can be expanded in terms of the characters $\mathbf{c h}_{m}=\operatorname{trace}\left(\mathbf{o}^{m}\right)$ of the representations $\mathbf{o}^{m}$; it turns out these have a simple explicit expression [9, p. 259]

$$
\mathbf{c h}_{m}(g)=\frac{\sin \left(\frac{(2 m+1)}{2} \theta(g)\right)}{\sin \left(\frac{\theta(g)}{2}\right)}
$$

and are thus class functions themselves. As a consequence, $\widehat{h}_{i, j}^{m}=\alpha_{m} \delta_{i, j}$ for some scalars $\alpha_{m}$. Consequently, 5.53) becomes

$$
Q(u)=\sum_{m}(2 m+1) \alpha_{m} \sum_{i, j}\left|\widehat{u}_{i, j}^{m}\right|^{2} .
$$

Therefore, it is sufficient to show that $\alpha_{m} \leq 0$ for all $m \geq 1$. They are given by

$$
\begin{aligned}
\alpha_{m} & =\frac{1}{2 m+1} \int_{\operatorname{SO}(3)} h(g) \mathbf{c h}_{m}(g) d g \\
& =\frac{1}{2 m+1} \int_{0}^{\pi} f(\theta) \frac{\sin \left(\frac{(2 m+1)}{2} \theta\right)}{\sin \left(\frac{\theta}{2}\right)} \frac{1-\cos \theta}{\pi} d \theta \quad \text { (by [9. p. 260]) } \\
& =\frac{1}{\pi(2 m+1)} \int_{0}^{\pi} f(\theta)(\cos (m \theta)-\cos ((m+1) \theta)) d \theta \\
& =-\frac{1}{\pi(2 m+1)} \int_{0}^{\pi} f^{\prime}(\theta)\left(\frac{\sin (m \theta)}{m}-\frac{\sin ((m+1) \theta)}{m+1}\right) d \theta
\end{aligned}
$$

where we integrated by parts at the last step. 
Define the function $\psi(m)$ for $m>0$ as

$$
\begin{aligned}
\psi(m)=\int_{0}^{\pi} f^{\prime}(\theta) \frac{\sin (m \theta)}{m} d \theta & =-\int_{0}^{\pi} f^{\prime \prime}(\theta) \frac{1-\cos (m \theta)}{m^{2}} d \theta \\
& =\frac{1}{m^{2} \theta_{*}} \int_{0}^{m \theta_{*}} \frac{1-\cos (t)}{t} d t,
\end{aligned}
$$

where we substituted in the specific form (5.44) of $f$. Using (5.57) in (5.56), we can express $\alpha_{m}$ as

$$
\alpha_{m}=\frac{1}{\pi(2 m+1)}(\psi(m+1)-\psi(m)) .
$$

We'll show that $\psi$ is a decreasing function for $m \geq 0$. First, compute

$$
\psi^{\prime}(m)=\frac{1}{m^{3} \theta_{*}}\left(\left[1-\cos \left(m \theta_{*}\right)\right]-2 \int_{0}^{m \theta_{*}} \frac{1-\cos (t)}{t} d t\right) .
$$

We'll show that $\psi^{\prime}(m) \leq 0$ for all $m \geq 0$. To that end, consider the function

$$
\phi(x)=[1-\cos (x)]-2 \int_{0}^{x} \frac{1-\cos (t)}{t} d t .
$$

It is sufficient to show that $\phi(x) \leq 0$ only for $x \in[0, \pi]$ since the integral in 5.60 is an increasing function of $x$ and the term $1-\cos (x)$ reaches its maximum at $x=\pi$. Taylor expanding, we get

$$
\phi(x)=\sum_{n=1}^{\infty}(-1)^{n+1} \frac{(n-1)}{n(2 n) !} x^{2 n} .
$$

When $x \in[0, \pi]$, the ratio between magnitudes of the $(n+1)^{\text {th }}$ and the $n^{\text {th }}$ terms in this alternating series is

$$
\frac{n^{2} x^{2}}{\left(n^{2}-1\right)(2 n+1)(2 n+2)} \leq \frac{2 \pi^{2}}{45}<1 \quad \text { for all } n \geq 2 .
$$

Therefore, $\phi(x) \leq 0$ for all $x \in[0, \pi]$. The foregoing discussion shows that this implies $\alpha_{m} \leq 0$ for all $m \geq 1$, establishing the conditional negative semidefiniteness of $\sigma_{i, j}$.

\subsection{Minimizing Movements Interpretation}

In Section 5.1, we exhibited Algorithms (5.7)-(5.8) and (5.26)-(5.29) as resulting from the simple, iterative optimization technique of repeatedly minimizing the linearization of the cost function over the constraint set. It is also possible to interpret them as implementing minimizing movements (as in, e.g., [2, 24]) on the energy $E_{\delta t}$ given in (4.5).

Indeed, it turns out that

$$
E_{\delta t}(u)-E_{\delta t}\left(u-u^{k}\right)=\mathcal{L}_{E_{\delta t}}\left(u^{k}, u\right)
$$


up to terms constant in $u$. Thus, minimizing $\mathcal{L}_{E_{\delta t}}\left(u^{k}, u\right)$, as Algorithm (5.7)- 5.8) does at the $k^{\text {th }}$ time step, is equivalent to one step of minimizing movements for $E_{\delta t}(u)$ with $-E_{\delta t}\left(u-u^{k}\right)$ as the movement limiting term. Note that when $E_{\delta t}(\cdot)$ is concave, as in the numerous cases identified in Section 5.2, the movement limiting term $-E_{\delta t}\left(u-u^{k}\right)$ is convex and therefore achieves its minimum value of 0 at $u=u^{k}$, as it should, ensuring the dissipation of $E_{\delta t}$ at every step; this observation constitutes an alternative proof of the unconditional stability of Algorithm (5.7)(5.8). The connection between $-E_{\delta t}\left(u-u_{k}\right)$ and the classical movement limiting term in [2,24], namely

$$
\frac{1}{\delta t} \int_{\Sigma \Delta \Sigma^{k}}\left|d_{\Sigma^{k}}\right| d x
$$

where $d_{\Sigma^{k}}$ denotes the signed distance function to $\Sigma^{k}$, can be motivated by considering the two-phase case where $\Sigma_{1}^{k}$ and $\Sigma_{2}^{k}$ are half-spaces

$$
\Sigma_{1}^{k}=\left\{x: x_{1} \leq 0\right\} \quad \text { and } \quad \Sigma_{2}^{k}=\left\{x: x_{1} \geq 0\right\}
$$

on the periodic domain $D=\left[-\frac{L}{2}, \frac{L}{2}\right)^{d}$ and $\Sigma_{1}$ is obtained from $\Sigma_{1}^{k}$ by moving $\partial \Sigma_{1}^{k}$ in the normal direction by $\delta$ :

$$
\Sigma_{1}=\left\{x: x_{1} \leq \delta\right\} \quad \text { and } \quad \Sigma_{2}=\left\{x: x_{1} \geq \delta\right\} .
$$

Observe that in this case

$$
\frac{1}{\delta t} \int_{\Sigma \Delta \Sigma^{k}}\left|d_{\Sigma^{k}}\right| d x=\frac{1}{\delta t} \frac{\delta^{2} L^{d-1}}{2} .
$$

Since we expect $\delta=O(\delta t)$ in a single step of Algorithm (5.7)-(5.8) wherever grain boundaries are smooth, we have $\delta \ll \sqrt{\delta t}$, which for the Gaussian kernel (3.1) implies

$$
\begin{aligned}
- & E_{\delta t}\left(\mathbb{1}_{\Sigma_{1}}-\mathbb{1}_{\Sigma_{1}^{k}}, \mathbb{1}_{\Sigma_{2}}-\mathbb{1}_{\Sigma_{2}^{k}}\right) \\
& =\frac{2 \sigma_{1,2}}{\sqrt{\delta t}} \int_{D}\left(\mathbb{1}_{\Sigma_{1}}-\mathbb{1}_{\Sigma_{1}^{k}}\right) G_{\delta t} *\left(\mathbb{1}_{\Sigma_{1}}-\mathbb{1}_{\Sigma_{1}^{k}}\right) d x \\
& =\frac{2 \sigma_{1,2}}{\sqrt{\delta t}} \int_{D}\left[G_{\delta t / 2} *\left(\mathbb{1}_{\Sigma_{1}}-\mathbb{1}_{\Sigma_{1}^{k}}\right)\right]^{2} d x \\
& \approx \frac{2 \sigma_{1,2}}{\sqrt{\delta t}} L^{d-1} \int_{-L / 2}^{L / 2} \delta^{2} \frac{1}{2 \pi(\delta t)} e^{-x_{1}^{2} /(\delta t)} d x_{1} \\
& \approx \frac{\sigma_{1,2}}{\delta t} \frac{\delta^{2} L^{d-1}}{\sqrt{\pi}} .
\end{aligned}
$$


Results of the Appendix indeed show that when the convolution kernel is the Gaussian 3.1) we have

$$
E_{\delta t}\left(\Sigma_{1}, \Sigma_{2}\right) \stackrel{\Gamma}{\rightarrow} \frac{\sigma_{1,2}}{\sqrt{\pi}}\left(\operatorname{Per}\left(\Sigma_{1}\right)+\operatorname{Per}\left(\Sigma_{2}\right)\right)=\frac{2 \sigma_{1,2}}{\sqrt{\pi}} \operatorname{Per}\left(\Sigma_{1}\right) .
$$

We thus see from (5.62) and (5.63) that $-E_{\delta t}\left(u-u^{k}, v-v^{k}\right)$ plays precisely the role of the movement limiter (5.61). This also explains why Algorithm (5.7)-(5.8) leads to the very specific mobilities $\mu_{i, j}=\frac{1}{\sigma_{i, j}}$.

In the case of Algorithm (5.26)-(5.29) for general mobilities, we have

$$
E_{\delta t}(u)+\left(-E_{\delta t}\left(u-u^{k}\right)+\sum_{i=1}^{N} \int u_{i} R_{i}^{k} d x\right)=\mathcal{L}_{F_{\delta t}}\left(u^{k}, u\right)
$$

up to terms constant in $u$, so that minimizing $\mathcal{L}_{F_{\delta t}}\left(u^{k}, u\right)$ at the $k^{\text {th }}$ time step is equivalent to carrying out one step of minimizing movements for $E_{\delta t}$, this time with the movement limiting term given in parentheses in (5.64). Note that each one of the additional terms $\int u_{i} R_{i}^{k} d x$ acts to limit movement, as it achieves its minimum value at $u_{i}=u_{i}^{k}$ as was noted previously in Section 5.2. There, interpretation of the $R_{i}^{k}$ in terms of the distance function was also already given.

\subsection{A Gauss-Seidel Version}

In this epilogue to Section 5, we describe a slightly more costly version of Algorithm (5.26) -5.29) that can be guaranteed to dissipate energy (4.2) for all triangle-inequality-satisfying surface tensions $\sigma \in \mathcal{T}_{N}$. It differs from Algorithm (5.26)-5.29) in computing the convolutions of the phases more frequently.

In words, at the $\ell^{\text {th }}$ inner step of Algorithm 5.65 - 5.68) (see p. 842), only those points belonging to phase $\ell+1$ are updated, in the sense that they are potentially assigned to one of the other $N-1$ phases. Immediately thereafter, all convolutions and retardation terms are refreshed. If the convolutions and retardation terms are refreshed per time step as opposed to per inner step, Algorithm (5.65)-(5.68) reduces to Algorithm (5.26)-(5.29) of Section 5.1.

Roughly speaking, the behavior of Algorithm (5.65)-(5.68) near a point $p \in$ $\Gamma_{i, j} \backslash \mathcal{J}_{\geq 3}$ (i.e., on a smooth interface between two phases, away from junctions) is the same as that of Algorithm 5.26-5.29). Indeed, it is reasonable to expect that if the normal speed of $\Gamma_{i, j}$ is nonzero near $p$, then the partition and the relevant retardation functions are updated near $p$ either at inner step $\ell=i$ or inner step $\ell=j$, but not at both, and certainly not at any $\ell \notin\{i, j\}$. But then Algorithm (5.65)-(5.68) agrees with Algorithm (5.26)-(5.29). Thus, the formal consistency argument offered for Algorithm (5.26)-(5.29) in Section 5.1 applies here as well. As for the stability of Algorithm (5.65)-(5.68), we have the following:

PROPOSITION 5.7. Algorithm 5.65-(5.68) is unconditionally gradient stable for all $\sigma \in \mathcal{T}_{N}$ : It dissipates energy (4.2) for all time step sizes $\delta t \geq 0$. 
Algorithm: Given the initial partition $\Sigma^{0}$ with $\Sigma_{i}^{0}=\left\{x: \psi_{i}^{0}(x)>0\right\}$, obtain the partition $\Sigma^{k+1}$ at time step $t=(\delta t)(k+1)$ from the partition $\Sigma^{k}$ at time $t=(\delta t) k$ using $N$ inner steps $\Sigma^{k, \ell}$, with $\ell=0,1, \ldots, N-1, \Sigma_{i}^{k, 0}=\Sigma_{i}^{k}$, and $\Sigma_{i}^{k, N-1}=\Sigma_{i}^{k+1}$.

Obtain $\Sigma^{k, \ell+1}$ from $\Sigma^{k, \ell}$ as follows:

1. Form the convolutions:

$$
\phi_{i}^{k, \ell}=G_{\delta t} *\left(\sum_{j=1}^{N} \sigma_{i, j} \mathbb{1}_{\Sigma_{j}^{k, \ell}}\right) .
$$

2. Form the retardation functions:

$$
R_{i}^{k, \ell}=\max _{j \neq i} \frac{1}{2}\left(1-\mu_{i, j} \sigma_{i, j}\right) \psi_{j}^{k, \ell} .
$$

3. Form the comparison functions

$$
\begin{aligned}
& \psi_{i}^{k, \ell+1}= \\
& \begin{cases}\max \left\{\psi_{i}^{k, \ell},\left(\min _{j \neq i} \phi_{j}^{k, \ell}+R_{j}^{k, \ell}\right)-\left(\phi_{i}^{k, \ell}+R_{i}^{k, \ell}\right)\right\} & \text { if } i \neq \ell+1, \\
\min \left\{\psi_{i}^{k, \ell},\left(\min _{j \neq i} \phi_{j}^{k, \ell}+R_{j}^{k, \ell}\right)-\left(\phi_{i}^{k, \ell}+R_{i}^{k, \ell}\right)\right\} & \text { if } i=\ell+1 .\end{cases}
\end{aligned}
$$

4. Threshold the comparison functions $\psi_{i}^{k, \ell+1}$ :

$$
\Sigma_{i}^{k, \ell+1}=\left\{x: \psi_{i}^{k, \ell+1}(x)>0\right\} .
$$

Proof of Proposition 5.7. At each time step, the $\ell^{\text {th }}$ inner step of the algorithm replaces some of the points belonging to phase $\ell+1$ with the rest. It is therefore sufficient to show that replacing phase 1 with phases $2,3, \ldots, N$ as the algorithm does decreases the energy. Such a perturbation can be written as

$$
u_{i}(x, t)= \begin{cases}u_{1}(x)-t \sum_{i \neq 1} \phi_{i}(x) & \text { if } i=1, \\ u_{i}(x)+t \phi_{i}(x) & \text { otherwise }\end{cases}
$$

where $\phi_{i}(x) \geq 0$ for all $x$ and $i=2,3, \ldots, N$.

Energy (4.5) turns out to be concave along such perturbation directions as long as $\sigma \in \mathcal{T}_{N}$ :

$$
\begin{aligned}
\frac{d^{2}}{d t^{2}} E_{\delta t}(u(t)) & =\sum_{i, j} \sigma_{i, j} \int\left(G_{\delta t} * \frac{d}{d t} u_{i}\right)\left(G_{\delta t} * \frac{d}{d t} u_{j}\right) d x \\
& =2 \sum_{i<j} \sigma_{i, j} \int\left(G_{\delta t} * \frac{d}{d t} u_{i}\right)\left(G_{\delta t} * \frac{d}{d t} u_{j}\right) d x=
\end{aligned}
$$




$$
\begin{aligned}
= & -2 \sum_{j=2}^{N} \sigma_{1, j} \int\left(\sum_{i \neq 1} \phi_{i} * G_{\delta t}\right)\left(\phi_{j} * G_{\delta t}\right) d x \\
& +2 \sum_{1<i<j} \sigma_{i, j} \int\left(\phi_{i} * G_{\delta t}\right)\left(\phi_{j} * G_{\delta t}\right) d x \\
= & -2 A+2 B
\end{aligned}
$$

where

$$
\begin{aligned}
A & =\sum_{\substack{i \neq 1 \\
j \neq 1}} \sigma_{1, j} \int\left(\phi_{i} * G_{\delta t}\right)\left(\phi_{j} * G_{\delta t}\right) d x, \\
B & =\sum_{1<i<j} \sigma_{i, j} \int\left(\phi_{i} * G_{\delta t}\right)\left(\phi_{j} * G_{\delta t}\right) d x
\end{aligned}
$$

Looking at these terms separately, we have

$$
\begin{aligned}
A= & \sum_{i} \sigma_{1, i}\left\|\phi_{i} * G_{\delta t}\right\|_{L^{2}}^{2}+\sum_{1<i<j} \sigma_{1, j} \int\left(\phi_{i} * G_{\delta t}\right)\left(\phi_{j} * G_{\delta t}\right) d x \\
& +\sum_{1<j<i} \sigma_{1, j} \int\left(\phi_{i} * G_{\delta t}\right)\left(\phi_{j} * G_{\delta t}\right) d x .
\end{aligned}
$$

Looking at $B$, using the triangle inequality

$$
\sigma_{i, j} \leq \sigma_{1, i}+\sigma_{1, j}
$$

and the fact

$$
\left(\phi_{i} * G_{\delta t}\right)(x) \geq 0 \text { for all } x \text { and } i \neq 1,
$$

we get

$$
\begin{aligned}
B \leq & \sum_{1<i<j} \sigma_{1, i} \int\left(\phi_{i} * G_{\delta t}\right)\left(\phi_{j} * G_{\delta t}\right) d x \\
& +\sum_{1<i<j} \sigma_{1, j} \int\left(\phi_{i} * G_{\delta t}\right)\left(\phi_{j} * G_{\delta t}\right) d x
\end{aligned}
$$

Putting $A$ and $B$ together gives

$$
\frac{d^{2}}{d t^{2}} E_{\delta t}(u(t)) \leq-2 \sum_{i} \sigma_{1, i}\left\|\phi_{i} * G_{\delta t}\right\|^{2} .
$$

Thus, $E_{\delta t}$ is concave in the perturbation directions that arise in Algorithm (5.65)(5.68). The algorithm seeks a minimum of the linearization of $E_{\delta t}$ in the space of these directions and therefore, by Lemma 5.2, decreases the energy at every time step. 


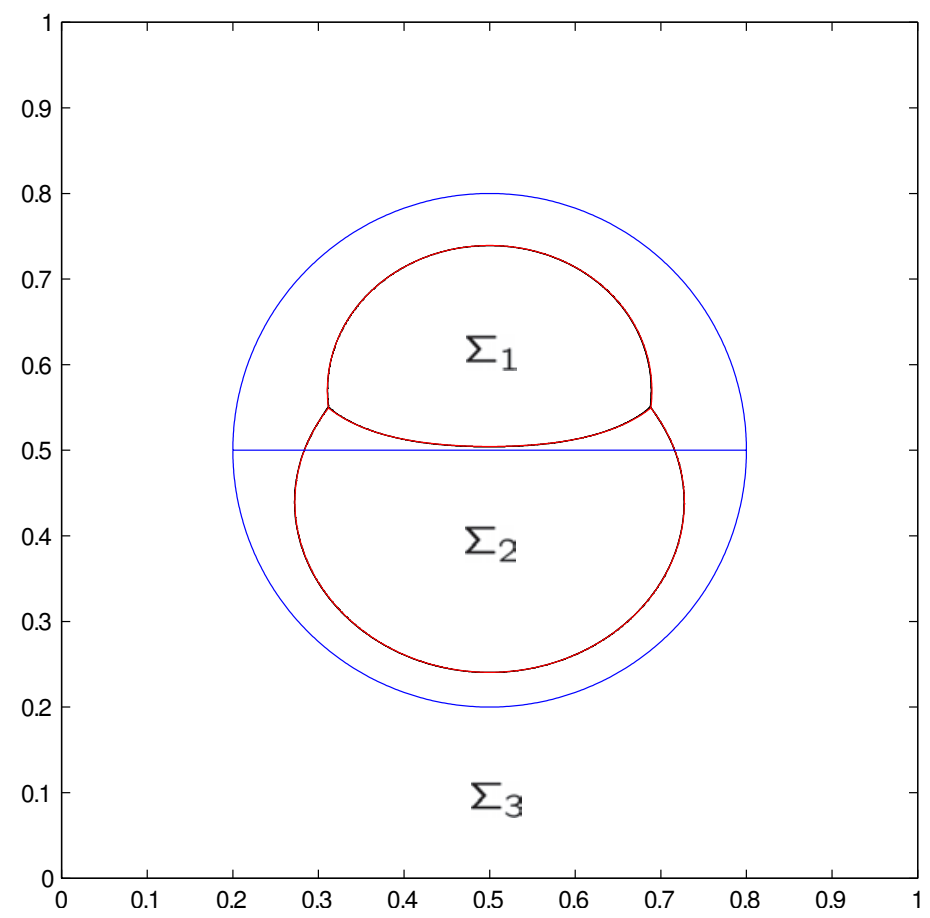

FIGURE 6.1. The blue curve shows the initial condition. Red curve shows the result of dynamics 1.5 and 1.7 computed using the proposed threshold dynamics algorithm of Section 5, with a $\left(90^{\circ}, 135^{\circ}\right.$, $135^{\circ}$ ) angle condition at the triple junction and all mobilities $\mu_{i, j}=1$. The black curve shows the same dynamics computed using front tracking. (The two curves are in excellent agreement and almost indistinguishable due to the line width of the plot).

\section{Numerical Evidence}

This section presents a variety of numerical tests of Algorithm (5.26)-(5.29) from Section 5. There are two types of test: (1) Classical numerical convergence studies for short-time evolution (during which topological changes do not take place) starting from an initial condition with triple junctions formed by the meeting of smooth curves, and (2) challenging configurations that involve topological changes, multiple junctions, nonembeddable surface tensions, wetting, and nucleation.

\subsection{Comparisons with Front Tracking}

In the absence of topological changes, and when starting from a smooth initial condition consisting only of triple junctions, a very appropriate and efficient algorithm for computing the curvature flow (1.5) and (1.7) is front tracking (see, e.g., [6]), especially in the plane. 
The initial condition in this set of experiments is shown in Figure 6.1 as the blue curve. It is evolved under dynamics 1.5 and 1.7 with surface tensions given by $\sigma_{1,2}=\sigma_{1,3}=1$ and $\sigma_{2,3}=\sqrt{2}$. The corresponding junction angles are $\left(\theta_{1}, \theta_{2}, \theta_{3}\right)=\left(90^{\circ}, 135^{\circ}, 135^{\circ}\right)$. The final configuration at time $t=0.0107$, computed using Algorithm (5.26)-(5.29) of Section 5 on a $3200 \times 3200$ grid, is shown as the red curve. The same configuration computed via front tracking is shown as the black curve. The table below shows the error as measured in the Hausdorff distance between the boundary $\partial \Sigma_{1}$ of phase $\Sigma_{1}$ computed using front tracking versus the proposed algorithm. All mobilities were $\mu_{i, j}=1$.

\begin{tabular}{|c|c|c|c|}
\hline \# Time steps & \# Grid points & Hausdorff dist. & Conv. rate \\
\hline 8 & $100 \times 100$ & 0.0678 & - \\
\hline 16 & $200 \times 200$ & 0.0339 & 1.00 \\
\hline 32 & $400 \times 400$ & 0.0174 & 0.962 \\
\hline 64 & $800 \times 800$ & 0.0082 & 1.09 \\
\hline 128 & $1600 \times 1600$ & 0.0040 & 1.04 \\
\hline 256 & $3200 \times 3200$ & 0.0018 & 1.15 \\
\hline
\end{tabular}

The same initial condition (blue curve in Figure 6.1) was used for testing Algorithm 5.26-5.29) with surface tensions $\sigma_{1,2}=\frac{5}{4}, \sigma_{1,3}=\frac{3}{2}$, and $\sigma_{2,3}=1$. The corresponding junction angles are $\left(\theta_{1}, \theta_{2}, \theta_{3}\right) \approx\left(138.6^{\circ}, 97.18^{\circ}, 124.2^{\circ}\right)$. The table below shows the error in phase 1, once again as measured in the Hausdorff distance between the front tracking solution and the solution obtained from Algorithm (5.26)-(5.29). All mobilities were $\mu_{i, j}=1$.

\begin{tabular}{|c|c|c|c|}
\hline \# Time steps & \# Grid points & Hausdorff dist. & Conv. rate \\
\hline 8 & $100 \times 100$ & 0.0806 & - \\
\hline 16 & $200 \times 200$ & 0.0392 & 1.04 \\
\hline 32 & $400 \times 400$ & 0.0185 & 1.08 \\
\hline 64 & $800 \times 800$ & 0.0092 & 1.01 \\
\hline 128 & $1600 \times 1600$ & 0.0044 & 1.06 \\
\hline 256 & $3200 \times 3200$ & 0.0019 & 1.21 \\
\hline
\end{tabular}

\subsection{Comparisons with Exact Solutions}

A well-known exact solution of dynamics $(1.5)$ and $(1.7)$ is the grim-reaper solution [16]. Here, two of the interfaces are traveling waves moving with constant vertical speed, while the third remains a line segment; see Figure 6.2. We will consider the following asymmetric case:

$$
\sigma=\left(\begin{array}{ccc}
0 & 1 & \frac{\sqrt{2}}{1+\sqrt{3}} \\
1 & 0 & \frac{2}{1+\sqrt{3}} \\
\frac{\sqrt{2}}{1+\sqrt{3}} & \frac{2}{1+\sqrt{3}} & 0
\end{array}\right),
$$




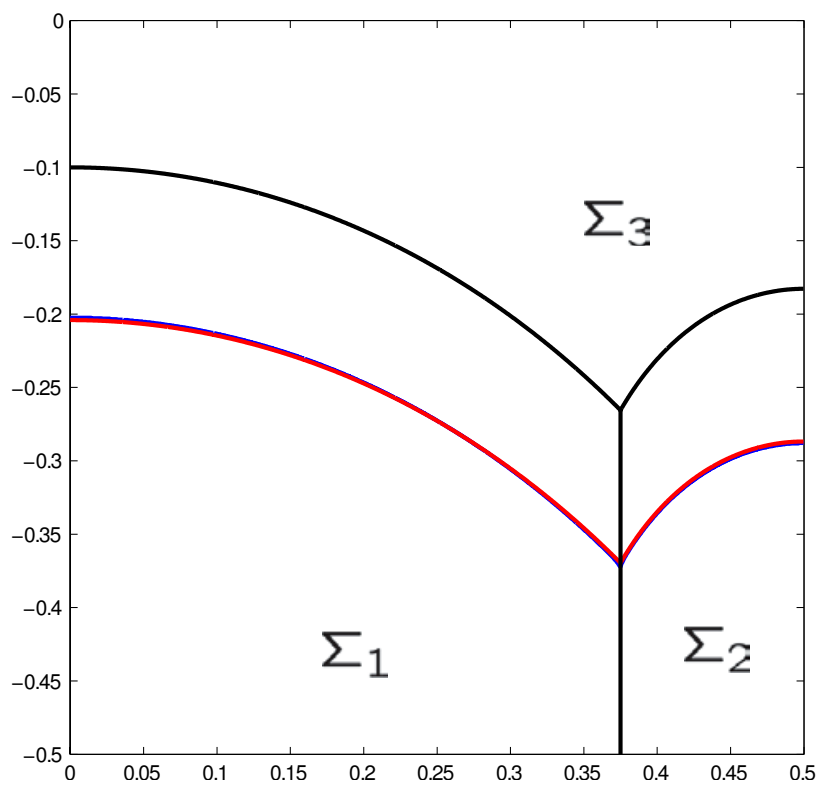

FIGURE 6.2. Numerical convergence test against an exact, travelling wave solution. The initial condition is the configuration of black curves. The blue curves are the computed solution (on a $1600 \times 1600$ grid) and the red ones are the exact solution, at time $t=0.096$ (they are in excellent agreement and almost indistinguishable). The angles at the junction are $\left(135^{\circ}, 150^{\circ}, 75^{\circ}\right)$.

and the mobilities are given by

$$
\mu_{1,2}=\mu_{1,3}=1 \quad \text { and } \quad \mu_{2,3}=\frac{1}{4 \sqrt{2}} .
$$

The corresponding angles at the junction are $\left(135^{\circ}, 150^{\circ}, 75^{\circ}\right)$. The two interfaces $\Gamma_{1,3}$ and $\Gamma_{2,3}$ are then graphs of functions $f_{1,3}(x, t):\left[0, \frac{3}{8}\right] \rightarrow \mathbb{R}$ and $f_{2,3}(x, t):$ $\left[\frac{3}{8}, \frac{1}{2}\right] \rightarrow \mathbb{R}$ that move by vertical translation:

$$
\begin{aligned}
& f_{1,3}(x, t)=\frac{3}{2 \pi} \log \left(\cos \left(\frac{2 \pi}{3} x\right)\right)-\frac{2 \sqrt{2} \pi}{3(1+\sqrt{3})} t \\
& f_{2,3}(x, t)=\frac{3}{8 \pi} \log \left(\frac{1}{2} \cos \left(\frac{4 \pi(1-2 x)}{3}\right)\right)-\frac{2 \sqrt{2} \pi}{3(1+\sqrt{3})} t .
\end{aligned}
$$

The interfaces satisfy the natural boundary condition of $90^{\circ}$ intersection with the boundary of the domain $\left[0, \frac{1}{2}\right] \times\left[-\frac{1}{2}, 0\right]$ (i.e., $\partial_{x} f_{1,3}(0, t)=0$ and $\partial_{x} f_{2,3}\left(\frac{1}{2}, t\right)=$ $0)$. Numerically, the initial configuration is extended evenly to $[0,1] \times[-1,0]$ by reflection, which is then computed with periodic boundary conditions using Algorithm 5.26-5.29. 


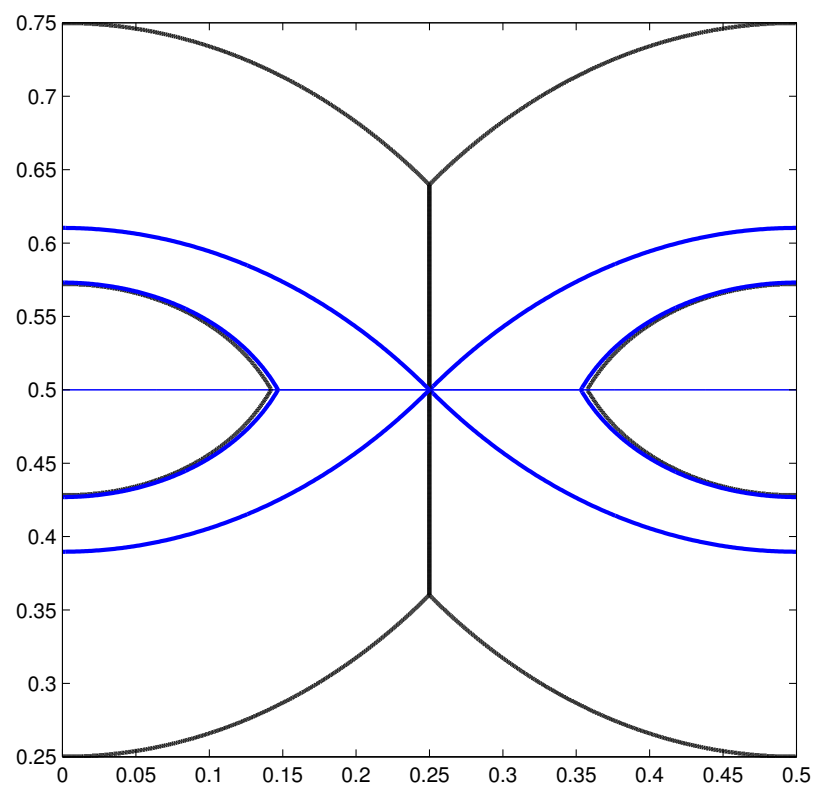

FIGURE 6.3. Comparison with front tracking on an example involving a topological change that is well understood. Two junctions collide, and then split off along an orthogonal path. Angles at the junction change from $\left(90^{\circ}, 135^{\circ}, 135^{\circ}\right)$ to $\left(120^{\circ}, 120^{\circ}, 120^{\circ}\right)$ before and after the topological event. Solution generated by Algorithm (5.26) -5.29 is in black (almost indistinguishable from the exact solution in blue).

The $L^{\infty}$ error between the computed and exact $f_{1,3}$ and $f_{2,3}$ at time $t=0.096$ is shown in the table below. Figure 6.2 shows the initial condition, the computed solution, and the exact solution in black, blue, and red, respectively.

\begin{tabular}{|c|c|c|c|}
\hline \# Time steps & \# Grid points & $L^{\infty}$ error & Conv. rate \\
\hline 192 & $100 \times 100$ & 0.0381 & - \\
\hline 384 & $200 \times 200$ & 0.0211 & 0.85 \\
\hline 768 & $400 \times 400$ & 0.0104 & 1.02 \\
\hline 1536 & $800 \times 800$ & 0.0061 & 0.77 \\
\hline 3072 & $1600 \times 1600$ & 0.0029 & 1.07 \\
\hline
\end{tabular}

\subsection{Topological Change}

Here we test the algorithm on a solution that goes through a topological change that is well understood. The initial condition is of "grim reaper" type, described previously in the previous subsection. Hence, the exact form of the solution (which serves as the benchmark) is known until the moment of topological change. At that critical time, two triple junctions collide, and by all accounts ought to split off immediately in a particular manner: As shown in Figure 6.3, the two junctions 
traveling towards each other vertically before the critical time should split off into two new junctions traveling horizontally away from each other immediately after the collision, forming a new horizontal interface between them. Beyond the critical time, we compute the benchmark solution using front tracking based on this expectation.

Define the profile

$$
\phi(x)=\frac{1}{\pi} \log (\cos (\pi x)) .
$$

The initial configuration is as follows:

$$
\begin{aligned}
& \Sigma_{1}^{0}=\left\{(x, y): y<\frac{1}{4}-\phi\left(\frac{1}{4}-\left|x-\frac{1}{4}\right|\right)\right\}, \\
& \Sigma_{2}^{0}=\left\{(x, y): x<\frac{1}{4} \text { and } \frac{1}{4}-\phi(x)<y<\frac{3}{4}+\phi(x)\right\}, \\
& \Sigma_{3}^{0}=\left\{(x, y): x>\frac{1}{4} \text { and } \frac{1}{4}-\phi(x)<y<\frac{3}{4}+\phi(x)\right\}, \\
& \Sigma_{4}^{0}=\left\{(x, y): y>\frac{3}{4}+\phi\left(\frac{1}{4}-\left|x-\frac{1}{4}\right|\right)\right\} .
\end{aligned}
$$

The surface tension matrix is

$$
\sigma=\left(\begin{array}{cccc}
0 & 1 & 1 & 1 \\
1 & 0 & \sqrt{2} & 1 \\
1 & \sqrt{2} & 0 & 1 \\
1 & 1 & 1 & 0
\end{array}\right)
$$

so that triple junctions of type $\left(\Sigma_{1}, \Sigma_{2}, \Sigma_{3}\right)$ and $\left(\Sigma_{2}, \Sigma_{3}, \Sigma_{4}\right)$ before the topological change have angles $\left(90^{\circ}, 135^{\circ}, 135^{\circ}\right)$ and $\left(135^{\circ}, 135^{\circ}, 90^{\circ}\right)$, respectively, and triple junctions of type $\left(\Sigma_{1}, \Sigma_{2}, \Sigma_{4}\right)$ and $\left(\Sigma_{1}, \Sigma_{3}, \Sigma_{4}\right)$ after the topological change have angles $\left(120^{\circ}, 120^{\circ}, 120^{\circ}\right)$. All mobilities were taken to be $\mu_{i, j}=\frac{1}{\sigma_{i, j}}$ in this example, so that all interfaces move with normal speed $\kappa$.

\subsection{Wetting}

Triangle inequality $(1.3)$ is not necessary for Algorithm (5.26) -5.29 ) to dissipate the approximate energy 4.2 , which presumably converges to the lower semicontinuous envelope of $(1.2)$ when $\sigma \notin \mathcal{T}_{N}$. Numerical experiments show that in these wetting cases, the algorithm can instantaneously nucleate a new phase along the boundary between two others, as might be expected. Figure 6.4 shows the evolution in a four-phase setting, starting from an initial configuration containing phases $\Sigma_{1}, \Sigma_{2}$, and $\Sigma_{3}$ only. The surface tensions are given by

$$
\sigma=\left(\begin{array}{cccc}
0 & \frac{3}{2} & 1 & \frac{1}{2} \\
\frac{3}{2} & 0 & 1 & \frac{1}{2} \\
1 & 1 & 0 & 1 \\
\frac{1}{2} & \frac{1}{2} & 1 & 0
\end{array}\right),
$$



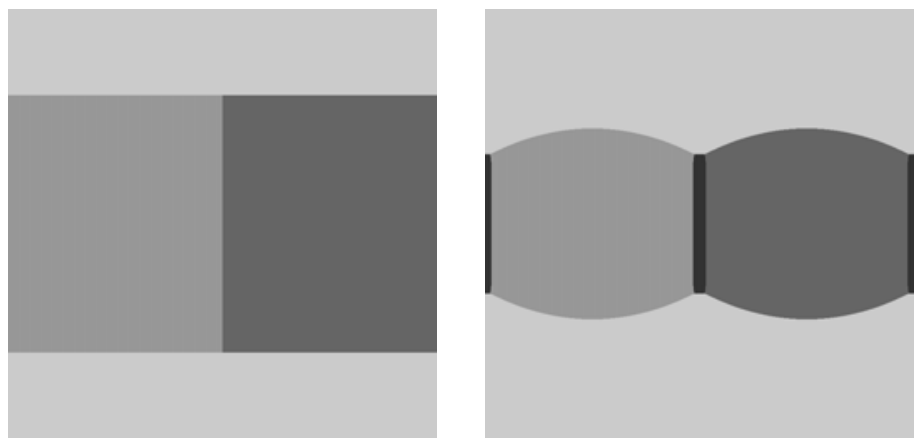

FIGURE 6.4. Example of wetting due to violation of the triangle inequality. Left: The initial condition contains only phases 1, 2, and 3 . The corresponding $3 \times 3$ submatrix of the full $4 \times 4$ surface tension matrix satisfies the triangle inequality, even though the full matrix does not. Right: Nevertheless, Algorithm (5.26)-(5.29) is aware of the possibility of a fourth phase, and immediately nucleates a thin layer of it along the $\Gamma_{1,2}$ interface present in the initial condition. That thin wetting layer of phase 4, shown as the darkest region, remains between phases 1 and 2 throughout the evolution. See also Figure 6.5 .

violating the triangle inequality: $\sigma_{1,4}+\sigma_{2,4}=1<\frac{3}{2}=\sigma_{1,2}$. Nevertheless, it turns out that $\sigma$ is conditionally negative semidefinite. Therefore, Corollary 5.4 applies, showing that Algorithm (5.26) -5.29) is unconditionally gradient stable for this ill-posed set of surface tensions.

The $3 \times 3$ submatrix $\sigma_{1: 3,1: 3}$ of 6.1 corresponding to the three phases present in the initial condition satisfies the triangle inequality. However, the algorithm is aware of the possibility of a fourth phase, and chooses to immediately nucleate a thin layer of it along the interface $\Gamma_{2,3}$, as can be seen in Figure 6.4. This thin layer of phase 4 remains between phases 1 and 2 throughout the evolution. Its thickness appears to depend on the time step size and scale as $\sqrt{\delta t}$.

The presence of a thin wetting layer of phase 4 between phases 1 and 2 reduces the effective cost of a transition between phase 1 and phase 2 down to $\sigma_{1,4}+$ $\sigma_{2,4}=1$ from $\sigma_{1,2}=\frac{3}{2}$. We would therefore expect the resulting dynamics to approximate flow with the surface tension matrix

$$
\sigma_{\text {effective }}=\left(\begin{array}{cccc}
0 & 1 & 1 & \frac{1}{2} \\
1 & 0 & 1 & \frac{1}{2} \\
1 & 1 & 0 & 1 \\
\frac{1}{2} & \frac{1}{2} & 1 & 0
\end{array}\right) .
$$

Indeed, Figure 6.5 compares the effective three-phase flow (with only phases 1, 2, and 3 present) computed using (6.2) versus the four-phase flow computed using the ill-posed set of surface tensions 6.1); it shows that the results are in fact very 


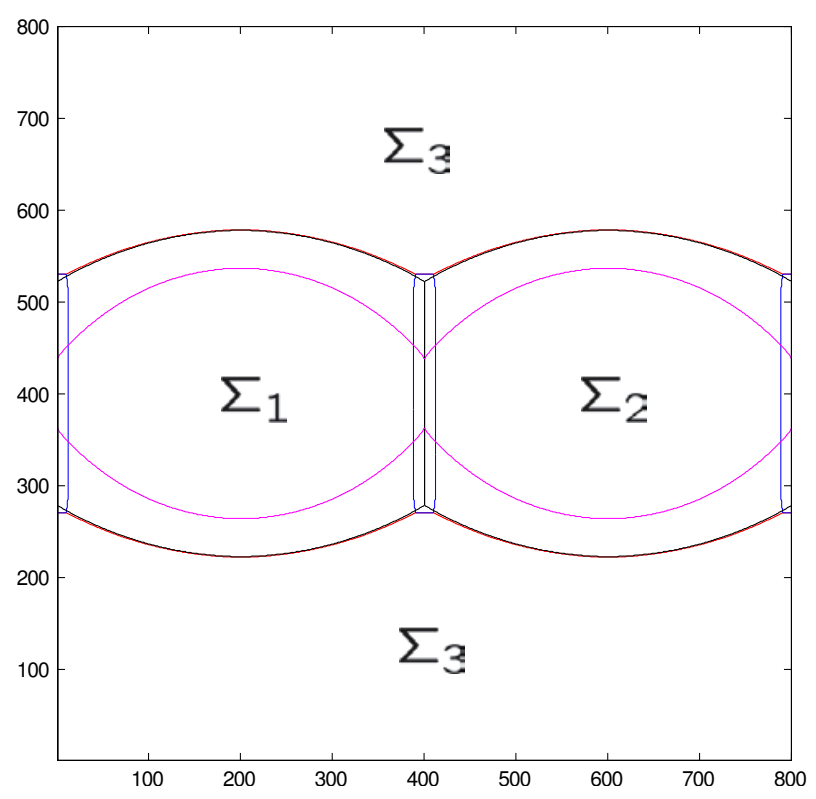

FIGURE 6.5. Comparison of the four-phase dynamics computed using Algorithm (5.26)- 5.29) with the effective three-phase dynamics when the surface tensions violate the triangle inequality, leading to wetting. The effective three-phase dynamics result is the black curve, while the red curve shows the result of the four-phase computation with the illposed surface tensions; they match very closely. The thin region indicated by the blue curve is the wetting layer automatically nucleated by Algorithm 5.26-5.29 during the four-phase computation. The magenta curve shows what the four-phase evolution would have been if the wetting layer had not been nucleated. See also Figure 6.4

close. In other words, Algorithm 5.26)-5.29) appears to capture the dynamics for the relaxation of model (1.2) when the model is ill-posed due to violation of the triangle inequality. Figure 6.5 also compares the computed dynamics with what the result would have been in the absence of a wetting layer, i.e., three-phase flow with surface tensions given by $\sigma_{1: 3,1: 3}$ in 6.1.

As pointed out in Section 5.1, nucleation can be prevented, if desired, without sacrificing the useful properties discussed in Section 5.2. Wetting would then take place only when phase 4 is present in the initial data and comes in contact with $\Gamma_{1,2}$ at some point during the evolution.

\subsection{Nucleation}

If the surface tension matrix $\sigma$ satisfies the triangle inequality (1.3) (i.e., $\sigma \in$ $\mathcal{T}_{N}$ ), wetting cannot occur. However, nucleation can still take place at junctions 

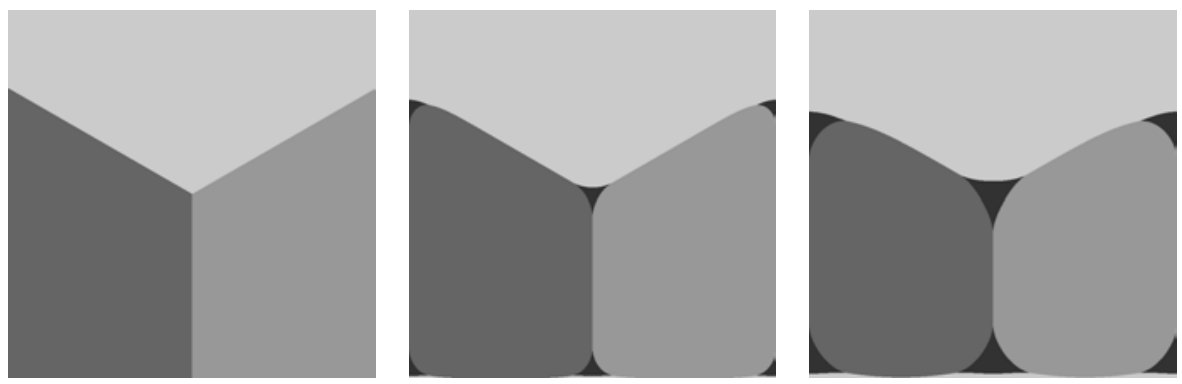

FIGURE 6.6. Nucleation and growth of a fourth phase in the evolution computed by Algorithm (5.26)-(5.29), even though the initial condition contains only three phases, and the surface tensions satisfy the triangle inequality. Leftmost panel shows the initial condition.

for certain $\sigma \in \mathcal{T}_{N}$. An example is the four-phase system with surface tension matrix

$$
\sigma(\varepsilon)=\left(\begin{array}{cccc}
0 & 1 & 1 & \frac{1}{2}+\varepsilon \\
1 & 0 & 1 & \frac{1}{2}+\varepsilon \\
1 & 1 & 0 & \frac{1}{2}+\varepsilon \\
\frac{1}{2}+\varepsilon & \frac{1}{2}+\varepsilon & \frac{1}{2}+\varepsilon & 0
\end{array}\right)
$$

which happens to be one of the several types of polyphase grain structures considered in [7]. We see that $\sigma(\varepsilon) \in \mathcal{T}_{4}$ for $\varepsilon \in\left(0, \frac{3}{2}\right)$, since then all surface tensions are within a factor of 2 of each other; hence, the model is well-posed and no wetting along a smooth interface $\Gamma_{i, j}$ can take place. Nevertheless, as explained in [7], when

$$
\varepsilon \in\left(0, \frac{2-\sqrt{3}}{4+2 \sqrt{3}}\right),
$$

a triple junction made up of phases 1,2 , and 3 cannot be stable: even when in its equilibrium configuration of symmetric, $120^{\circ}$ junction angles, it would be unstable under the nucleation of phase 4.

Figure 6.6 shows this taking place during a four-phase simulation using Algorithm (5.26)-(5.29), where the surface tensions were taken as in 6.3) with $\varepsilon=$ 0.03 . Note that once again the algorithm is unconditionally gradient stable for this set of surface tensions.

\section{Appendix: Convergence of the Energies}

In this section, we show that the multiphase interfacial energy functional (1.2) can be obtained as the $\Gamma$-limit of the quadratic nonlocal energy (4.2). We establish this result for isotropic surface energies, but just under the assumption that the surface tension matrix $\sigma$ satisfies the triangle inequality (1.3): $\sigma \in \mathcal{T}_{N}$. In particular, our result yields the well-known lower semicontinuity of the limiting 
functional by a new and elementary argument, relying on an approximate monotonicity of the approximating functional; see Section A.1. The other ingredients to our $\Gamma$-convergence result can be found in the literature [1, 28].

For the results on lower semicontinuity in the case of anisotropic surface tensions, we refer to the sufficient criteria of Bi-convexity [3, 2.2] and (B)-convexity [3, 2.3] for $B V$-ellipticity [3, 2.1], that is equivalent to lower semicontinuity [3, Theorem 2.1]. We also refer to [29] for the sufficient criteria of B2-convexity, which turns out to be equivalent to the triangle inequality in the isotropic case.

At least for the purposes of this section, we may take the convolution kernel $G$ to be more general than merely the Gaussian. We require it have the form $G_{\epsilon}(x)=$ $\epsilon^{-d} G\left(\frac{x}{\epsilon}\right)$, where the mask $G(\hat{x})$ is smooth and satisfies

$$
\begin{array}{cc}
G \geq 0, \quad \int_{\mathbb{R}^{d}} G d \hat{x}=1, & \int_{\mathbb{R}^{d}}|\hat{x}| G d \hat{x}<\infty, \\
G=G(|\hat{x}|), \quad|\hat{\nabla} G(\hat{x})| \lesssim G\left(\frac{\hat{x}}{2}\right), \quad \hat{\nabla} G(\hat{x}) \cdot \hat{x} \leq 0 .
\end{array}
$$

Recall that we say a configuration $u$ is admissible if it respects (4.4). In this section, we take $D=[0, L)^{d} \subset \mathbb{R}^{d}$ and assume that all $u_{i}$ are $L$-periodic in every coordinate.

Proposition A.1. Suppose that for an admissible sequence of configurations $\left\{u_{\epsilon}\right\}_{\epsilon \downarrow 0},\left\{E_{\epsilon}\left(u_{\epsilon}\right)\right\}_{\epsilon \downarrow 0}$ is bounded. Then $\left\{u_{\epsilon}\right\}_{\epsilon \downarrow}$ precompact in $L^{1}\left([0, L)^{d}\right)$.

Under the (strong) topology of $L^{1}\left([0, L)^{d}\right)$, the sequence of functionals $\left\{E_{\epsilon}\right\} \downarrow 0$ $\Gamma$-converges to the functional $E$ defined on the set of admissible u's given by

$$
\begin{aligned}
E(u):=c_{0} \sum_{i, j} \sigma_{i j} \frac{1}{2} & \left(\int_{[0, L)^{d}}\left|\nabla u_{i}\right|+\int_{[0, L)^{d}}\left|\nabla u_{j}\right|\right. \\
& \left.-\int_{[0, L)^{d}}\left|\nabla\left(u_{i}+u_{j}\right)\right|\right),
\end{aligned}
$$

if $u_{i} \in B V\left([0, L)^{d},\{0,1\}\right)$ for all $i=1,2, \ldots, N$, and $E(u)=+\infty$ otherwise. Here $c_{0}:=\frac{\left|B^{d-1}\right|}{\left|\mathbb{S}^{d-1}\right|} \int_{\mathbb{R}^{d}}|\hat{x}| G d \hat{x}$.

Note that $\frac{1}{2}\left(\int_{[0, L)^{d}}\left|\nabla u_{i}\right|+\int_{[0, L)^{d}}\left|\nabla u_{j}\right|-\int_{[0, L)^{d}}\left|\nabla\left(u_{i}+u_{j}\right)\right|\right)$ formally is the $(d-1)$-dimensional measure of the interface between $\left\{u_{i}=1\right\}$ and $\left\{u_{j}=1\right\}$ on the "torus" $[0, L)^{d}$.

Proof of Proposition A.1. The precompactness statement is a consequence of Lemma A.4 of Section A.3.

We turn to the recovery sequence for a given admissible $u$. If $u_{i} \in B V\left([0, L)^{d}\right.$, $\{0,1\}$ ) for all $i=1,2, \ldots, N$, Lemma A.3 shows that we may take $u$ itself as a recovery sequence. If this is not the case, then Lemma A.4 implies that $E_{\epsilon}(u) \uparrow$ 
$\infty=E(u)$ as $\epsilon \downarrow 0$ so that also in this case, we may take $u$ itself as a recovery sequence.

We finally turn to the lower semicontinuity part of $\Gamma$-convergence. Given is an admissible sequence $\left\{u_{\epsilon}\right\}_{\epsilon \downarrow 0}$ that converges to an admissible $u$ in $L^{1}\left([0, L)^{d}\right)$. According to Lemma A.2 we have for any $\epsilon_{0}>0$

$$
E_{\epsilon}\left(u_{\epsilon}\right) \geq\left(\frac{\epsilon_{0}}{\epsilon_{0}+\epsilon}\right)^{d+1} E_{\epsilon_{0}}\left(u_{\epsilon}\right) .
$$

Furthermore, since for fixed $\epsilon_{0}>0, E_{\epsilon_{0}}$ is continuous with respect to $L^{1}\left([0, L)^{d}\right)$ (on the space of admissible configurations), we have

$$
\lim _{\epsilon \downarrow 0} E_{\epsilon_{0}}\left(u_{\epsilon}\right)=E_{\epsilon_{0}}(u) .
$$

Both statements combine to

$$
\liminf _{\epsilon \downarrow 0} E_{\epsilon}\left(u_{\epsilon}\right) \geq E_{\epsilon_{0}}(u)
$$

for all $\epsilon_{0}>0$. Finally, the same argument as in case of the recovery sequence yields

$$
\lim _{\epsilon_{0} \downarrow 0} E_{\epsilon_{0}}(u)=E(u) \in[0, \infty] .
$$

The two last statements combine into the desired

$$
\liminf _{\epsilon \downarrow 0} E_{\epsilon}\left(u_{\epsilon}\right) \geq E(u) .
$$

\section{A.1 Approximate Monotonicity}

This subsection establishes an approximate sense of monotonicity for the functionals 4.5): they are approximately increasing as the width of the convolution kernel decreases. This might be the only novel ingredient of our discussion of $\Gamma$-convergence of 4.5.

Lemma A.2. Suppose $u$ is admissible. Then we have for all $0<\epsilon \leq \epsilon_{0}$ :

$$
E_{\epsilon}(u) \geq\left(\frac{\epsilon_{0}}{\epsilon_{0}+\epsilon}\right)^{d+1} E_{\epsilon_{0}}(u) .
$$

Proof of Lemma A.2. We fix $u$ and $\epsilon>0$. We first argue that A.3 is a consequence of the following two statements:

$$
E_{\epsilon}(u) \geq E_{N \epsilon}(u) \text { for all } N \in \mathbb{N}
$$

and

$$
\epsilon^{\prime d+1} E_{\epsilon^{\prime}}(u) \geq \epsilon^{d+1} E_{\epsilon}(u) \text { for all } \epsilon^{\prime} \geq \epsilon .
$$

Indeed, for $\epsilon_{0} \geq \epsilon$ let $N \in \mathbb{N}$ be such that

$$
(N-1) \epsilon<\epsilon_{0} \leq N \epsilon ; \quad \text { in particular, } \quad \frac{\epsilon_{0}}{N \epsilon} \geq \frac{\epsilon_{0}}{\epsilon_{0}+\epsilon} .
$$


We obtain $\mathrm{A} .3$ as follows:

$$
\begin{aligned}
E_{\epsilon}(u) \stackrel{\text { A.4 }}{\geq} E_{N \epsilon}(u)=(N \epsilon)^{-(d+1)}(N \epsilon)^{d+1} E_{N \epsilon}(u) \\
\stackrel{\text { A.5), A.6 }}{\geq}(N \epsilon)^{-(d+1)} \epsilon_{0}^{d+1} E_{\epsilon_{0}}(u) \stackrel{\text { A.6 }}{\geq}\left(\frac{\epsilon_{0}}{\epsilon_{0}+\epsilon}\right)^{d+1} E_{\epsilon_{0}}(u) .
\end{aligned}
$$

Before addressing the main ingredient (A.4), we turn to the easy ingredient (A.5), which can be reformulated as

$$
\frac{d}{d \epsilon} E_{\epsilon}(u) \geq-\frac{d+1}{\epsilon} E_{\epsilon}(u) .
$$

As we shall see, this is a consequence of the radial monotonicity of $G$, cf. A.1. To ease notation, we introduce

$$
F(h):=\sum_{i, j} \sigma_{i j} \int u_{i}(x) u_{j}(x+h) d x=\sum_{i \neq j} \sigma_{i j} \int u_{i}(x) u_{j}(x+h) d x
$$

and note that

$$
E_{\epsilon}(u)=\frac{1}{\epsilon} \int G_{\epsilon}(h) F(-h) d h=\int \frac{1}{\epsilon^{d+1}} G\left(\frac{h}{\epsilon}\right) F(-h) d h .
$$

Hence we obtain (A.7) as follows:

$$
\begin{aligned}
\frac{d}{d \epsilon} E_{\epsilon}(u) & \stackrel{\text { A.9. }}{=} \int\left(-\frac{1}{\epsilon^{d+2}}\right)\left((d+1) G\left(\frac{h}{\epsilon}\right)+\hat{\nabla} G\left(\frac{h}{\epsilon}\right) \cdot \frac{h}{\epsilon}\right) F(-h) d h \\
& \stackrel{\text { A. } 11}{\geq} \int\left(-\frac{1}{\epsilon^{d+2}}\right)(d+1) G\left(\frac{h}{\epsilon}\right) F(-h) d h \stackrel{\text { A.9 }}{=}-\frac{d+1}{\epsilon} E_{\epsilon}(u) .
\end{aligned}
$$

We now turn to the proof of (A.4). We start by arguing that for (A.4), it is enough to show that

$$
F\left(h+h^{\prime}\right) \leq F(h)+F\left(h^{\prime}\right) \text { for all } h, h^{\prime} \in \mathbb{R}^{d} .
$$

Indeed, on the one hand, A.10 can be iterated to yield

$$
F(N h) \leq N F(h) \quad \text { for all } h \in \mathbb{R}^{d}, N \in \mathbb{N} .
$$

On the other hand, we have by A.9

$$
E_{\epsilon}(u)=\frac{1}{\epsilon} \int G_{\epsilon}(h) F(-h) d h=\frac{1}{\epsilon} \int G(\hat{h}) F(-\epsilon \hat{h}) d \hat{h} .
$$

We thus obtain, using the nonnegativity of $G$,

$$
\begin{aligned}
E_{N \epsilon}(u) \stackrel{\text { A.12 }}{=} \frac{1}{N \epsilon} \int G(\hat{h}) F(-N \epsilon h) d \hat{h} \\
\stackrel{\text { A.11 }}{\leq} \frac{1}{\epsilon} \int G(\hat{h}) F(-\epsilon h) d \hat{h} \stackrel{\text { A.12 }}{=} E_{\epsilon}(u) .
\end{aligned}
$$


We now turn to A.10]. We write for abbreviation $x^{\prime}:=x+h, x^{\prime \prime}:=x^{\prime}+h^{\prime}$, and note that for $i \neq j$ we have

$$
\begin{aligned}
& u_{i}(x) u_{j}\left(x^{\prime \prime}\right)-u_{i}(x) u_{j}\left(x^{\prime}\right)-u_{i}\left(x^{\prime}\right) u_{j}\left(x^{\prime \prime}\right) \\
& \stackrel{\text { [4.4] }}{=} u_{i}(x) \sum_{k} u_{k}\left(x^{\prime}\right) u_{j}\left(x^{\prime \prime}\right)-u_{i}(x) u_{j}\left(x^{\prime}\right) \sum_{k} u_{k}\left(x^{\prime \prime}\right) \\
& \quad-\sum_{k} u_{k}(x) u_{i}\left(x^{\prime}\right) u_{j}\left(x^{\prime \prime}\right) \\
& =\sum_{k}\left(u_{i}(x) u_{k}\left(x^{\prime}\right) u_{j}\left(x^{\prime \prime}\right)-u_{i}(x) u_{j}\left(x^{\prime}\right) u_{k}\left(x^{\prime \prime}\right)-u_{k}(x) u_{i}\left(x^{\prime}\right) u_{j}\left(x^{\prime \prime}\right)\right) .
\end{aligned}
$$

We observe that the contribution from $k \in\{i, j\}$ to this sum has a sign:

$$
\begin{aligned}
& u_{i}(x) u_{i}\left(x^{\prime}\right) u_{j}\left(x^{\prime \prime}\right)-u_{i}(x) u_{j}\left(x^{\prime}\right) u_{i}\left(x^{\prime \prime}\right)-u_{i}(x) u_{i}\left(x^{\prime}\right) u_{j}\left(x^{\prime \prime}\right) \\
& +u_{i}(x) u_{j}\left(x^{\prime}\right) u_{j}\left(x^{\prime \prime}\right)-u_{i}(x) u_{j}\left(x^{\prime}\right) u_{j}\left(x^{\prime \prime}\right)-u_{j}(x) u_{i}\left(x^{\prime}\right) u_{j}\left(x^{\prime \prime}\right) \\
& =-u_{i}(x) u_{j}\left(x^{\prime}\right) u_{i}\left(x^{\prime \prime}\right)-u_{j}(x) u_{i}\left(x^{\prime}\right) u_{j}\left(x^{\prime \prime}\right) \stackrel{[4.4}{\leq} 0 .
\end{aligned}
$$

Hence we obtain

$$
\begin{aligned}
& u_{i}(x) u_{j}\left(x+h+h^{\prime}\right)-u_{i}(x) u_{j}(x+h)-u_{i}(x+h) u_{j}\left(x+h+h^{\prime}\right) \leq \\
& \sum_{k \neq i, j}\left(u_{i}(x) u_{k}\left(x^{\prime}\right) u_{j}\left(x^{\prime \prime}\right)-u_{i}(x) u_{j}\left(x^{\prime}\right) u_{k}\left(x^{\prime \prime}\right)-u_{k}(x) u_{i}\left(x^{\prime}\right) u_{j}\left(x^{\prime \prime}\right)\right)
\end{aligned}
$$

Multiplying both sides by $\sigma_{i, j}$, integrating over $x \in[0, L)^{d}$, using translation invariance, and summing over all pairs $(i, j)$ with $i \neq j$ yields by definition (A.8) of $F$ :

$$
\begin{array}{r}
F\left(h+h^{\prime}\right)-F(h)-F\left(h^{\prime}\right) \\
\leq \sum_{\substack{i, j, k \\
\text { pairwise different }}} \sigma_{i j} \int u_{i}(x) u_{k}(x+h) u_{j}\left(x+h+h^{\prime}\right) \\
-u_{i}(x) u_{j}(x+h) u_{k}\left(x+h+h^{\prime}\right) \\
-u_{k}(x) u_{i}(x+h) u_{j}\left(x+h+h^{\prime}\right) d x .
\end{array}
$$


We now claim that the right-hand side of $\mathrm{A} .13$ has a sign. Indeed, using the triangle inequality for the $\sigma_{i j}$ 's, the integrand can be estimated as follows:

$$
\begin{gathered}
\sum_{\substack{i, j, k \\
\text { pairwise different } \\
[1.3],[4.4}} \sigma_{i j}\left(u_{i}(x) u_{k}\left(x^{\prime}\right) u_{j}\left(x^{\prime \prime}\right)-u_{i}(x) u_{j}\left(x^{\prime}\right) u_{k}\left(x^{\prime \prime}\right)-u_{k}(x) u_{i}\left(x^{\prime}\right) u_{j}\left(x^{\prime \prime}\right)\right) \\
\sum_{\begin{array}{c}
i, j, k \\
\text { pairwise different }
\end{array}}\left(\sigma_{i k} u_{i}(x) u_{k}\left(x^{\prime}\right) u_{j}\left(x^{\prime \prime}\right)+\sigma_{k j} u_{i}(x) u_{k}\left(x^{\prime}\right) u_{j}\left(x^{\prime \prime}\right)\right. \\
\\
\left.-\sigma_{i j} u_{i}(x) u_{j}\left(x^{\prime}\right) u_{k}\left(x^{\prime \prime}\right)-\sigma_{i j} u_{k}(x) u_{i}\left(x^{\prime}\right) u_{j}\left(x^{\prime \prime}\right)\right) .
\end{gathered}
$$

Relabeling the indices, we see that the four contributions to the right-hand side cancel after summation: namely, the first term in the summand cancels with the third, and the second cancels with the fourth.

\section{A.2 Consistency}

The following lemma can essentially be found in [28][theorem 3.1]. We display our own proof, since we allow for a slightly more general convolution kernel $G$, and since our argument uses even less geometric measure theory (i.e., does not use the notion and regularity of reduced boundary).

Lemma A.3. Suppose $u$ is admissible in the sense of (4.4) and that, in addition, $u_{i} \in B V\left([0, L)^{d},\{0,1\}\right)$ for every $i=1,2, \ldots, N$. Then

$$
\lim _{\epsilon \downarrow 0} E_{\epsilon}(u)=c_{0} \sum_{i, j} \sigma_{i j} \frac{1}{2}\left(\int_{[0, L)^{d}}\left|\nabla u_{i}\right|+\int_{[0, L)^{d}}\left|\nabla u_{j}\right|-\int_{[0, L)^{d}}\left|\nabla\left(u_{i}+u_{j}\right)\right|\right)
$$

where $c_{0}:=\frac{\left|B^{d-1}\right|}{\left|\mathbb{S}^{d-1}\right|} \int_{\mathbb{R}^{d}}|\hat{x}| G d \hat{x}$.

Proof of Lemma A.3. The statement obviously reduces to

$$
\begin{aligned}
\lim _{\epsilon \downarrow 0} \frac{1}{\epsilon} \int_{[0, L)^{d}} \tilde{v} G_{\epsilon} * v d x \\
=\left|B^{d-1}\right| \int_{0}^{\infty} r^{d} G(r) d r \\
\quad \times \frac{1}{2}\left(\int_{[0, L)^{d}}|\nabla v|+\int_{[0, L)^{d}}|\nabla \widetilde{v}|-\int_{[0, L)^{d}}|\nabla(v+\widetilde{v})|\right),
\end{aligned}
$$

where $v \in B V\left([0, L)^{d},\{0,1\}\right)$ (which plays the role of $\left.u_{i}\right)$ and $\tilde{v} \in B V\left([0, L)^{d}\right.$, $\{0,1\}$ ) (which plays the role of $u_{j}$ ) satisfy

$$
v \tilde{v}=0 \quad \text { a.e. }
$$


The first reduction is to get rid of $G$ and to reduce the statement (A.14) to

$$
\begin{aligned}
& \lim _{\epsilon \downarrow 0} \frac{1}{\epsilon} \int_{\mathbb{S}^{d-1}} \int_{[0, L)^{d}} \tilde{v}(x) v(x+\epsilon \xi) d x d \xi= \\
& \left|B^{d-1}\right| \frac{1}{2}\left(\int_{[0, L)^{d}}|\nabla v|+\int_{[0, L)^{d}}|\nabla \widetilde{v}|-\int_{[0, L)^{d}}|\nabla(v+\widetilde{v})|\right) .
\end{aligned}
$$

Indeed, because of the radial symmetry of $G$, i.e., $G(x)=G(|x|)$ (cf. (A.1)), we have

$$
\begin{aligned}
\frac{1}{\epsilon} \int_{[0, L)^{d}} \tilde{v} G_{\epsilon} * v d x & =\frac{1}{\epsilon} \int_{\mathbb{R}^{d}} G(h) \int_{[0, L)^{d}} \tilde{v}(x) v(x+\epsilon h) d x d h \\
& =\int_{0}^{\infty} G(r) r^{d} \frac{1}{\epsilon r} \int_{\mathbb{S}^{d-1}} \int_{[0, L)^{d}} \widetilde{v}(x) v(x+\epsilon r \xi) d x d \xi d r .
\end{aligned}
$$

We thus see that (A.16) formally yields (A.14) by substituting for $\epsilon$ by $\epsilon r$ in A.16 and integrating with respect to the nonnegative measure $G(r) r^{d} d r$. We note that this measure is finite because of our moment assumption on $G$; cf. (A.1). We now make this argument rigorous by an application of Lebesgue's dominated convergence theorem: The dominating function is obtained as follows:

$$
\begin{aligned}
& \left|\frac{1}{\epsilon r} \int_{\mathbb{S}^{d-1}} \int_{[0, L)^{d}} \tilde{v}(x) v(x+\epsilon r \xi) d x d \xi\right| \\
& \stackrel{[\mathrm{A.15}]}{-}\left|\frac{1}{\epsilon r} \int_{\mathbb{S}^{d-1}} \int_{[0, L)^{d}} \tilde{v}(x)(v(x+\epsilon r \xi)-v(x)) d x d \xi\right| \\
& \quad \leq \frac{1}{\epsilon r} \int_{\mathbb{S}^{d-1}} \int_{[0, L)^{d}}|v(x+\epsilon r \xi)-v(x)| d x d \xi \\
& \quad \leq\left|\mathbb{S}^{d-1}\right| \int_{[0, L)^{d}}|\nabla v| .
\end{aligned}
$$

The second reduction step is to disintegrate $\mathbb{S}^{d-1}$ into individual axes $\pm \xi \in$ $\mathbb{S}^{d-1}$, which means to reduce (A.16) to

$$
\begin{aligned}
& \text { (A.18) } \lim _{\epsilon \downarrow 0} \frac{1}{\epsilon} \int_{[0, L)^{d}} \widetilde{v}(x)(v(x+\epsilon \xi)+v(x-\epsilon \xi)) d x= \\
& \frac{1}{2}\left(\int_{[0, L)^{d}}|\xi \cdot \nabla v|+\int_{[0, L)^{d}}|\xi \cdot \nabla \widetilde{v}|-\int_{[0, L)^{d}}|\xi \cdot \nabla(v+\widetilde{v})|\right),
\end{aligned}
$$


where $|\xi \cdot \nabla v|=|\xi \cdot v||\nabla v|$ with $v=\frac{\nabla v}{|\nabla v|}$ denoting the measure-theoretic normal (which exists by Besicovitch differentiation of measures). Formally, A.16 is obtained from A.18 by integration with respect to $\frac{1}{2} d \xi$. This is obvious for the left-hand side. For the right-hand side we note that because of symmetry,

$$
\int_{\mathbb{S}^{d-1}} \int_{[0, L)^{d}}|\xi \cdot \nabla v| d \xi=\int_{[0, L)^{d}} \int_{\mathbb{S}^{d-1}}|\xi \cdot v| d \xi|\nabla v|=\int_{\mathbb{S}^{d-1}}\left|\xi \cdot e_{d}\right| d \xi \int_{[0, L)^{d}}|\nabla v|
$$

where $e_{d}=(0, \ldots, 0,1)^{\top}$, and observe that for $s=x \cdot e_{d}$

$$
\begin{aligned}
\int_{\mathbb{S}^{d-1}}\left|\xi \cdot e_{d}\right| d \xi & =\int_{(-1,1)}|s|\left(\left|\mathbb{S}^{d-2}\right|{\sqrt{1-s^{2}}}^{d-2}\right) \sqrt{1-s^{2}} d s \\
& =\left|\mathbb{S}^{d-2}\right| \int_{0}^{\pi}|\cos \theta| \sin ^{d-2} \theta d \theta \\
& =2\left|\mathbb{S}^{d-2}\right| \int_{0}^{\pi / 2} \cos \theta \sin ^{d-2} \theta d \theta \\
& =2\left|\mathbb{S}^{d-2}\right| \int_{0}^{\pi / 2} \frac{d}{d \theta}\left[\frac{1}{d-1} \sin ^{d-1} \theta\right] d \theta \\
& =\left|\mathbb{S}^{d-2}\right| \frac{2}{d-1}=2\left|B^{d-1}\right| .
\end{aligned}
$$

To make this rigorous, we use once more dominated convergence based on the estimate

$$
\left|\frac{1}{\epsilon} \int_{[0, L)^{d}} \tilde{v}(x)(v(x+\epsilon \xi)+v(x-\epsilon \xi)) d x\right| \leq 2 \int|\nabla v|,
$$

which is obtained as A.17).

The third reduction step is to reduce $(\mathrm{A} .18]$ to the analogous statement for a single space dimension, namely: For any $w, \widetilde{w} \in B V([0, L),\{0,1\})$ with $w \widetilde{w}=0$ a.e., we have

$$
\begin{aligned}
& \text { (A.19) } \lim _{\epsilon \downarrow 0} \frac{1}{\epsilon} \int_{[0, L)}(w(s+\epsilon)+w(s-\epsilon)) \tilde{w}(s) d s= \\
& \frac{1}{2}\left(\int_{[0, L)}\left|\frac{d w}{d s}\right|+\int_{[0, L)}\left|\frac{d \tilde{w}}{d s}\right|-\int_{[0, L)}\left|\frac{d(w+\tilde{w})}{d s}\right|\right) .
\end{aligned}
$$

Indeed, by symmetry, it suffices to show A.18 for $\xi=e_{d}$, which prompts us to introduce the coordinates $s=e_{d} \cdot x$ and $x^{\prime}=x-s e_{d}$. We claim that (A.18) is 
obtained from applying A.19 to $w=w_{x^{\prime}}=v\left(x^{\prime}, \cdot\right)$ and $\widetilde{w}=\widetilde{w}_{x^{\prime}}=\widetilde{v}\left(x^{\prime}, \cdot\right)$ and formally integrating over $x^{\prime} \in[0, L)^{d-1}$ (with respect to the Lebesgue measure). This is (formally) obvious for the left-hand side of (A.19). For the right-hand side it follows from elementary BV-theory [14]: For any $v \in B V\left([0, L)_{x}^{d}\right)$ we have $w_{x^{\prime}} \in B V\left([0, L)_{s}\right)$ for a. e. $x^{\prime} \in[0, L)^{d-1}$ and

$$
\int_{[0, L)^{d-1}} \int_{[0, L)}\left|\frac{d w_{x^{\prime}}}{d s}\right| d x^{\prime}=\int_{[0, L)^{d}}\left|e_{d} \cdot \nabla v\right| .
$$

Again, the formal integration is made rigorous with the help of the principle of dominated convergence based on the estimate

$$
\mid \frac{1}{\epsilon} \int_{[0, L)} \widetilde{w}_{x^{\prime}}(s)\left(w_{x^{\prime}}(s+\epsilon)+w_{x^{\prime}}(s-\epsilon) d s\left|\leq 2 \int_{[0, L)}\right| \frac{d w_{x^{\prime}}}{d s} \mid,\right.
$$

and noting that the dominating function $\int_{[0, L)}\left|\frac{d w_{x^{\prime}}}{d s}\right|$ is integrable:

$$
\int_{[0, L)^{d-1}} \int_{[0, L)}\left|\frac{d w_{x^{\prime}}}{d s}\right| d x^{\prime}=\int_{[0, L)^{d}}\left|e_{d} \cdot \nabla v\right|<\infty .
$$

It remains to justify A.19]. Because of $w, \widetilde{w} \in B V([0, L),\{0,1\}), w$ and $\widetilde{w}$ are functions of $s$ that have a finite number of jumps between 0 and 1 . Let us denote the (pairwise different) jump points by $s_{1}, \ldots, s_{M}$ and $\tilde{s}_{1}, \ldots, \widetilde{s}_{\tilde{M}}$. Clearly,

$$
M=\int_{[0, L)}\left|\frac{d w}{d s}\right| \text { and } \tilde{M}=\int_{[0, L)}\left|\frac{d \tilde{w}}{d s}\right| \text {. }
$$

Because of $w \tilde{w}=0, w+\widetilde{w}$ jumps where either $w$ or $\tilde{w}$ jumps, so that

$$
\int_{[0, L)}\left|\frac{d(w+\tilde{w})}{d s}\right|=M+\tilde{M}-2 \#\left(\left\{s_{1}, \ldots, s_{M}\right\} \cap\left\{\widetilde{s}_{1}, \ldots, \tilde{s}_{\tilde{M}}\right\}\right) .
$$

Hence the right-hand side of A.19] is given by $\#\left(\left\{s_{1}, \ldots, s_{M}\right\} \cap\left\{\widetilde{s}_{1}, \ldots, \widetilde{s}_{\tilde{M}}\right\}\right)$. It thus remains to argue that

$$
\begin{aligned}
\lim _{\epsilon \downarrow 0} \frac{1}{\epsilon} \int_{[0, L)} \tilde{w}(s)(w(s+\epsilon)+w(s-\epsilon)) d s= & \\
& \#\left(\left\{s_{1}, \ldots, s_{M}\right\} \cap\left\{\tilde{s}_{1}, \ldots, \tilde{s}_{\tilde{M}}\right\}\right) .
\end{aligned}
$$

Indeed, it is elementary to see that if $\epsilon$ is smaller than any distance between two different elements of $\left\{\widetilde{s}_{1}, \ldots, \widetilde{s}_{\tilde{M}}\right\}$, we have exact equality:

$$
\frac{1}{\epsilon} \int_{[0, L)} \tilde{w}(s)(w(s+\epsilon)+w(s-\epsilon)) d s=\#\left(\left\{s_{1}, \ldots, s_{M}\right\} \cap\left\{\tilde{s}_{1}, \ldots, \tilde{s}_{\tilde{M}}\right\}\right),
$$

completing the proof. 


\section{A.3 Compactness}

The following lemma can essentially be found in [1, theorem 3.1]. For the convenience of the reader, we include its proof in our situation, following the lines of [1, theorem 3.1].

LemmA A.4. Suppose $\left\{u_{\epsilon}\right\}_{\epsilon \downarrow 0}$ is a sequence of admissible configurations such that $\left\{E_{\epsilon}\left(u_{\epsilon}\right)\right\}_{\epsilon \downarrow 0}$ is bounded. Then $\left\{u_{\epsilon}\right\}_{\epsilon \downarrow 0}$ is precompact in $L^{1}\left([0, L)^{d}\right)$. In addition, any accumulation point $u$ satisfies $u_{i} \in B V\left([0, L)^{d},\{0,1\}\right)$ for all $i=$ $1,2, \ldots, N$.

Proof of Lemma A.4. We note that for any fixed $i=1, \ldots, N$

$$
\begin{aligned}
& E_{\epsilon}\left(u_{\epsilon}\right) \geq\left(\min _{j \neq i} \sigma_{i j}\right) \frac{1}{\epsilon} \sum_{j \neq i} \int_{[0, L)^{d}} u_{j, \epsilon} G_{\epsilon} * u_{i, \epsilon} d x \\
& \stackrel{[4.4]}{=}\left(\min _{j \neq i} \sigma_{i j}\right) \frac{1}{\epsilon} \int_{[0, L)^{d}}\left(1-u_{i, \epsilon}\right) G_{\epsilon} * u_{i, \epsilon} d x .
\end{aligned}
$$

Writing $v_{\epsilon}:=u_{i, \epsilon} \in[0,1]$ for abbreviation, we hence have by (4.4)

$$
\frac{1}{\epsilon} \int_{[0, L)^{d}}\left(1-v_{\epsilon}\right) G_{\epsilon} * v_{\epsilon} d x \quad \text { stays bounded as } \epsilon \downarrow 0,
$$

and we want to show that $\left\{v_{\epsilon}\right\}_{\epsilon \downarrow 0}$ is precompact in $L^{1}\left([0, L)^{d}\right)$ and that any accumulation point $v$ is in $B V\left([0, L)^{d},\{0,1\}\right)$.

We start off by establishing several functional inequalities for an arbitrary $L$ periodic function $v(x) \in[0,1]$ :

$$
\int_{\mathbb{R}^{d}} G_{\epsilon}(h) \int_{[0, L)^{d}}|v(x+h)-v(x)| d x d h \leq 2 \int_{[0, L)^{d}}(1-v) G_{\epsilon} * v d x,
$$

$$
\begin{gathered}
\int_{[0, L)^{d}}\left|G_{\epsilon} * v-v\right| d x \leq \int_{\mathbb{R}^{d}} G_{\epsilon}(h) \int_{[0, L)^{d}}|v(x+h)-v(x)| d x d h, \\
\int_{[0, L)^{d}} v(1-v) d x \leq \int_{[0, L)^{d}}(1-v) G_{\epsilon} * v d x+\int_{[0, L)^{d}}\left|G_{\epsilon} * v-v\right| d x, \\
\int_{[0, L)^{d}}\left|\nabla\left(G_{\epsilon} * v\right)\right| d x \lesssim \epsilon^{-1} \int_{\mathbb{R}^{d}} G_{2 \epsilon}(h) \int_{[0, L)^{d}}|v(x+h)-v(x)| d x .
\end{gathered}
$$


We start with inequality (A.21). Because of $G(-h)=G(h)$ (cf. A.1), we have in particular

$$
\begin{aligned}
& \int_{[0, L)^{d}}(1-v) G_{\epsilon} * v d x \\
& =\int_{\mathbb{R}^{d}} G_{\epsilon}(h) \int_{[0, L)^{d}} v(x+h)(1-v(x)) d x d h \\
& =\frac{1}{2} \int_{\mathbb{R}^{d}} G_{\epsilon}(h) \int_{[0, L)^{d}} v(x+h)(1-v(x))+v(x)(1-v(x+h)) d x d h .
\end{aligned}
$$

Now A.21 follows since if we write $v=v(x) \in[0,1]$ and $v^{\prime}=v(x+h) \in[0,1]$, we have

$$
\left|v^{\prime}-v\right| \leq\left|v^{\prime}-v v^{\prime}\right|+\left|v v^{\prime}-v\right|=v^{\prime}(1-v)+v\left(1-v^{\prime}\right) .
$$

Inequality A.22 follows from Jensen's inequality using $G_{\epsilon} \geq 0, \int G_{\epsilon} d h=1$, cf. (A.1). Inequality A.23) follows from the fact that if we write $v=v(x) \in[0,1]$ and $v^{\prime}=\left(G_{\epsilon} * v\right)(x) \in[0,1]$, we have

$$
v(1-v) \leq v^{\prime}(1-v)+\left|v^{\prime}-v\right| \text {. }
$$

We now turn to A.24. We note that

$$
\nabla\left(G_{\epsilon} * v\right)(x)=\int_{\mathbb{R}^{d}} \nabla G_{\epsilon}(h) v(x+h) d h=\int_{\mathbb{R}^{d}} \nabla G_{\epsilon}(h)(v(x+h)-v(x)) d h,
$$

so that

$$
\int_{[0, L)^{d}}\left|\nabla\left(G_{\epsilon} * v\right)\right| d x \leq \int_{\mathbb{R}^{d}}\left|\nabla G_{\epsilon}(h)\right| \int_{[0, L)^{d}}|v(x+h)-v(x)| d x d h .
$$

We observe that because of (A.1) we have

$$
\left|\nabla G_{\epsilon}(h)\right|=\epsilon^{-d-1}|\nabla G|\left(\frac{h}{\epsilon}\right) \lesssim \epsilon^{-d-1} G\left(\frac{h}{2 \epsilon}\right) \sim \epsilon^{-1} G_{2 \epsilon}(h) .
$$

Inserting A.26 into A.25 yields A.24).

We now may conclude: By A.20, (A.21), and A.24), $\nabla\left(G_{\epsilon} * v_{\epsilon}\right)$ is bounded in $L^{1}\left([0, L)^{d}\right)$. Since in addition $G_{\epsilon} * v_{\epsilon} \in[0,1], G_{\epsilon} * v_{\epsilon}$ is precompact in $L^{1}\left([0, L)^{d}\right)$. In view of A.20, A.21, and A.22, $G_{\epsilon} * v_{\epsilon}-v_{\epsilon}$ converges to 0 in $L^{1}\left([0, L)^{d}\right)$. Hence $v_{\epsilon}$ is also precompact in $L^{1}\left([0, L)^{d}\right)$, as desired. Now let $v$ be any accumulation point of $v_{\epsilon}$ in $L^{1}\left([0, L)^{d}\right)$. Then it is also an accumulation point of $G_{\epsilon} * v_{\epsilon}$. Since $\nabla\left(G_{\epsilon} * v_{\epsilon}\right)$ is bounded in $L^{1}\left([0, L)^{d}\right), v$ is in $B V\left([0, L)^{d}\right)$. Finally, $v \in\{0,1\}$, as can be seen by using in A.23 the inequality A.20 and the fact $G_{\epsilon} * v_{\epsilon}-v_{\epsilon} \rightarrow 0$ in $L^{1}$ that was noted above. 
Acknowledgment. This project started while the authors were visiting the Institute for Mathematics and its Applications and the University of Minnesota. Further work on the project was made possible through several visits to the Max Planck Institute for Mathematics in the Sciences and the University of Michigan. It's a pleasure to acknowledge the hospitality of these institutions. Selim Esedoglu was supported by National Science Foundation Grants DMS-0748333 and DMS0713767.

\section{Bibliography}

[1] Alberti, G.; Bellettini, G. A non-local anisotropic model for phase transitions: asymptotic behaviour of rescaled energies. European J. Appl. Math. 9 (1998), no. 3, 261-284. doi:10.1017/S0956792598003453

[2] Almgren, F.; Taylor, J. E.; Wang, L. Curvature-driven flows: a variational approach. SIAM J. Control Optim. 31 (1993), no. 2, 387-438. doi:10.1137/0331020

[3] Ambrosio, L.; Braides, A. Functionals defined on partitions in sets of finite perimeter. II. Semicontinuity, relaxation and homogenization. J. Math. Pures Appl. (9) 69 (1990), no. 3, 307-333.

[4] Avis, D.; Deza, M. The cut cone, $L^{1}$ embeddability, complexity, and multicommodity flows. Networks 21 (1991), no. 6, 595-617. doi:10.1002/net.3230210602

[5] Barles, G.; Georgelin, C. A simple proof of convergence for an approximation scheme for computing motions by mean curvature. SIAM J. Numer. Anal. 32 (1995), no. 2, 484-500. doi: $10.1137 / 0732020$

[6] Bronsard, L.; Wetton, B. T. R. A numerical method for tracking curve networks moving with curvature motion. J. Comput. Phys. 120 (1995), no. 1, 66-87. doi:10.1006/jcph.1995.1149

[7] Cahn, J. W. Stability, microstructural evolution, grain growth, and coarsening in a twodimensional two-phase microstructure. Acta Metallurgica et Materialia 39 (1991), no. 10, 2189-2199. doi:10.1016/0956-7151(91)90001-H

[8] Deza, M. M.; Laurent, M. Geometry of cuts and metrics. Algorithms and Combinatorics, 15. Springer, Berlin, 1997.

[9] Dym, H.; McKean, H. P. Fourier series and integrals. Probability and Mathematical Statistics, 14. Academic Press, New York-London, 1972.

[10] Elsey, M.; Esedoğlu, S.; Smereka, P. Diffusion generated motion for grain growth in two and three dimensions. J. Comput. Phys. 228 (2009), no. 21, 8015-8033. doi:10.1016/j.jcp.2009.07.020

[11] Elsey, M.; Esedoḡu, S.; Smereka, P. Large-scale simulation of normal grain growth via diffusion-generated motion. Proc. R. Soc. Lond. Ser. A Math. Phys. Eng. Sci. 467 (2011), no. 2126, 381-401. doi:10.1098/rspa.2010.0194

[12] Elsey, M.; Esedoglu, S.; Smereka, P. Large-scale simulations and parameter study for a simple recrystallization model. Philosophical Magazine 91 (2011), no. 11, 1607-1642. doi:10.1080/14786435.2010.546377

[13] Evans, L. C. Convergence of an algorithm for mean curvature motion. Indiana Univ. Math. J. 42 (1993), no. 2, 533-557. doi:10.1512/iumj.1993.42.42024

[14] Evans, L. C.; Gariepy, R. F. Measure theory and fine properties of functions. Studies in Advanced Mathematics. CRC Press, Boca Raton, Fla., 1992.

[15] Garcke, H.; Haas, R. Modeling of nonisothermal multi-component, multi-phase systems with convection. Mathematics 11, University of Regensburg, 2008.

[16] Garcke, H.; Nestler, B.; Stoth, B. A multiphase field concept: numerical simulations of moving phase boundaries and multiple junctions. Phase transformations in multicomponent melts, 325338. Wiley-VCH, Weinheim-Berlin, 2009. doi:10.1002/9783527624041.ch20 
[17] Haas, R. Modeling and analysis for general non-isothermal convective phase field systems. Thesis, University of Regensburg, 2007.

[18] Herring, C. Surface tension as a motivation for sintering. Fundamental contributions to the continuum theory of evolving phase interfaces in solids, 33-69. Springer, Berlin, 1999.

[19] Holm, E. A.; Hassold, G. N.; Miodownik, M. A. On misorientation distribution evolution during anisotropic grain growth. Acta Materialia 49 (2001), no. 15, 2981-2991. doi:10.1016/S13596454(01)00207-5

[20] Humphreys, F. J.; Hatherly, M. Recrystallization and related annealing phenomena. Second edition. Elsevier, Oxford, 2003.

[21] Janssens, K. G. F.; Raabe, D.; Kozeschnik, E.; Miodownik, M. A.; Nestler, B. Computational materials engineering: an introduction to microstructure evolution. Academic Press, Burlington, Mass.-San Diego-London, 2007.

[22] Kinderlehrer, D.; Livshits, I.; Rohrer, G. S.; Taasan, S.; Yu, P. Mesoscale simulation of the evolution of the grain boundary character distribution. Materials Science Forum 467 (2004), 1063-1068.

[23] Kinderlehrer, D.; Livshits, I.; Ta'asan, S. A variational approach to modeling and simulation of grain growth. SIAM J. Sci. Comput. 28 (2006), no. 5, 1694-1715 (electronic). doi: $10.1137 / 030601971$

[24] Luckhaus, S.; Sturzenhecker, T. Implicit time discretization for the mean curvature flow equation. Calc. Var. Partial Differential Equations 3 (1995), no. 2, 253-271. doi:10.1007/BF01205007

[25] Mascarenhas, P. Diffusion generated motion by mean curvature. CAM Report 92-33, University of California, Los Angeles, 1992. Available at: ftp://ftp.math.ucla.edu/pub/ camreport/cam92-33.pdf

[26] Merriman, B.; Bence, J. K.; Osher, S. J. Diffusion generated motion by mean curvature. Proceedings of the Computational Crystal Growers Workshop, 73-83. Edited by J. Taylor. American Mathematical Society, Providence, R.I., 1992.

[27] Merriman, B.; Bence, J. K.; Osher, S. Motion of multiple junctions: a level set approach. J. Comp. Phys. 112 (1994), no. 2, 334-363. doi:10.1006/jcph.1994.1105

[28] Miranda, M., Jr.; Pallara, D.; Paronetto, F.; Preunkert, M. Short-time heat flow and functions of bounded variation in $\mathbf{R}^{N}$. Ann. Fac. Sci. Toulouse Math. (6) 16 (2007), no. 1, 125-145. doi:10.5802/afst.1142

[29] Morgan, F. Lowersemicontinuity of energy clusters. Proc. Roy. Soc. Edinburgh Sect. A 127 (1997), no. 4, 819-822. doi:10.1017/S0308210500023842

[30] Mullins, W. W. Two-dimensional motion of idealized grain boundaries. J. Appl. Phys. 27 (1956), 900-904.

[31] Mumford, D.; Shah, J. Optimal approximations by piecewise smooth functions and associated variational problems. Comm. Pure Appl. Math. 42 (1989), no. 5, 577-685. doi:10.1002/cpa.3160420503

[32] Read, W. T.; Shockley, W. Dislocation models of crystal grain boundaries. Physical Review 78 (1950), no. 3, 275-289. doi:10.1103/PhysRev.78.275

[33] Ruuth, S. J. A diffusion-generated approach to multiphase motion. J. Comput. Phys. 145 (1998), no. 1, 166-192. doi:10.1006/jcph.1998.6028

[34] Ruuth, S. J. Efficient algorithms for diffusion-generated motion by mean curvature. J. Comput. Phys. 144 (1998), no. 2, 603-625. doi:10.1006/jcph.1998.6025

[35] Saye, R. I.; Sethian, J. A. The Voronoi implicit interface method for computing multiphase physics. Proc. Natl. Acad. Sci. USA 108 (2011), no. 49, 19498-19503. doi:10.1073/pnas.1111557108

[36] Saye, R. I.; Sethian, J. A. Analysis and applications of the Voronoi implicit interface method. J. Comput. Phys. 231 (2012), no. 18, 6051-6085. doi:10.1016/j.jcp.2012.04.004 
[37] Schoenberg, I. J. Metric spaces and positive definite functions. Trans. Amer. Math. Soc. 44 (1938), no. 3, 522-536. doi:10.2307/1989894

[38] Slepčev, D. Coarsening in nonlocal interfacial systems. SIAM J. Math. Anal. 40 (2008), no. 3, 1029-1048. doi:10.1137/080713598

[39] Topaz, C. M.; Bertozzi, A. L.; Lewis, M. A. A nonlocal continuum model for biological aggregation. Bull. Math. Biol. 68 (2006), no. 7, 1601-1623. doi:10.1007/s11538-006-9088-6

[40] Vilenkin, N. Ja. Special functions and the theory of group representations. Translations of Mathematical Monographs, 22. American Mathematical Society, Providence, R.I., 1968.

[41] Zhao, H.-K.; Chan, T.; Merriman, B.; Osher, S. A variational level set approach to multiphase motion. J. Comput. Phys. 127 (1996), no. 1, 179-195. doi:10.1006/jcph.1996.0167

\section{SELIM ESEDOḠLU}

Department of Mathematics

University of Michigan

530 Church St.

Ann Arbor, MI 48109

USA

E-mail: esedoglu@umich.edu

\section{Felix OTTO}

Max Planck Institute for Mathematics in the Sciences

Inselstraße 22

04103 Leipzig

GERMANY

E-mail: otto@mis.mpg.de

Received January 2013. 